\title{
Quantum Monte Carlo study of quasi-one-dimensional Bose gases
}

\author{
G. E. Astrakharchik ${ }^{(1,2)}$, D. Blume ${ }^{(3)}$, S. Giorgini ${ }^{(1)}$, and B. E. Granger ${ }^{(4)}$ \\ ${ }^{(1)}$ Dipartimento di Fisica, Università di Trento and BEC-INFM, I-38050 Povo, Italy \\ ${ }^{(2)}$ Institute of Spectroscopy, 142190 Troitsk, Moscow region, Russia \\ ${ }^{(3)}$ Department of Physics, Washington State University, Pullman, Washington 99164-2814, USA \\ ${ }^{(4)}$ Department of Physics, Santa Clara University, Santa Clara, California 95053, USA
}

(Dated: November 26, 2018)

\begin{abstract}
We study the behavior of quasi-one-dimensional (quasi-1d) Bose gases by Monte Carlo techniques, i.e., by the variational Monte Carlo, the diffusion Monte Carlo, and the fixed-node diffusion Monte Carlo technique. Our calculations confirm and extend our results of an earlier study [Astrakharchik et al., cond-mat/0308585. We find that a quasi-1d Bose gas i) is well described by a $1 \mathrm{~d}$ model Hamiltonian with contact interactions and renormalized coupling constant; ii) reaches the TonksGirardeau regime for a critical value of the $3 \mathrm{~d}$ scattering length $a_{3 d}$; iii) enters a unitary regime for $\left|a_{3 d}\right| \rightarrow \infty$, where the properties of the gas are independent of $a_{3 d}$ and are similar to those of a $1 \mathrm{~d}$ gas of hard-rods; and iv) becomes unstable against cluster formation for a critical value of the $1 \mathrm{~d}$ gas parameter. The accuracy and implications of our results are discussed in detail.

PACS numbers:
\end{abstract}

\section{INTRODUCTION}

Quasi-1d Bose gases have been realized in highlyelongated traps by tightly confining the transverse motion of the atoms to their zero point oscillations [1]. As compared to the $3 \mathrm{~d}$ case, the role of quantum fluctuations is enhanced in $1 \mathrm{~d}$ and these systems are predicted to exhibit peculiar properties, which cannot be described using traditional mean-field theories, but require more advanced many-body approaches. Particularly intriguing is the strong coupling regime, where, due to repulsion between particles, the quasi-1d Bose gas behaves as if it consisted of fictitious spinless fermions. This regime, the so called Tonks-Girardeau (TG) gas [2, 3, 4, 5], has not been achieved yet, but is one of the main focus areas of present experimental investigations in this field [6]. An interesting possibility to approach the strongly correlated TG regime is provided by magnetic field induced atom-atom Feshbach resonances [7, 8]. By utilizing this technique one can tune the $3 \mathrm{~d} s$-wave scattering length $a_{3 d}$, and hence the strength of atom-atom interactions, to essentially any value, including zero and $\pm \infty$.

Degenerate quantum gases near a Feshbach resonance have recently received a great deal of interest both experimentally and theoretically. At resonance $\left(\left|a_{3 d}\right| \rightarrow \infty\right)$ the $3 \mathrm{~d}$ scattering cross-section $\sigma$ is fixed by the unitary condition, $\sigma=4 \pi / k^{2}$, where $k$ is the relative wave vector of the two atoms. In this regime it is predicted that, if the range $r_{0}$ of the atom-atom interaction potential is much smaller than the average interparticle distance, the behavior of the gas is universal, i.e., independent of the details of the interatomic potential and independent of the actual value of $a_{3 d}[9,[10]$. This is known as the unitary regime 11. In the case of $3 \mathrm{~d}$ Bose gases, this unitary regime can most likely not be realized in experiments since three-body recombination is expected to set in when $a_{3 d}$ becomes comparable to the average interparticle distance. Three-body recombination leads to cluster formation and hence makes the gas-like state unstable. The situation is different for Fermi gases, for which the unitary regime has already been reached experimentally [8]. In this case, the Fermi pressure stabilizes the system even for large $\left|a_{3 d}\right|$.

In quasi-1d geometries a new length scale becomes relevant, namely, the oscillator length $a_{\rho}=\sqrt{\hbar /\left(m \omega_{\rho}\right)}$ of the tightly-confined transverse motion, where $m$ is the mass of the atoms and $\omega_{\rho}$ is the angular frequency of the harmonic trapping potential. For $\left|a_{3 d}\right| \gg a_{\rho}$, the gas is expected to exhibit a universal behavior if the range $r_{0}$ of the atom-atom interaction potential is much smaller than $a_{\rho}$ and the mean interparticle distance is much larger than $a_{\rho}$. It has been predicted that three-body recombination processes are suppressed for strongly interacting 1d Bose gases [12]. These studies raise the question whether the unitary regime can be reached in Bose gases confined in highly-elongated traps, that is, whether the quasi-1d bosonic gas-like state is stable against cluster formation as $a_{3 d} \rightarrow \pm \infty$.

This paper, which is an extension of an earlier study [13], investigates the properties of a quasi-1d Bose gas at zero temperature over a wide range of values of the $3 \mathrm{~d}$ scattering length $a_{3 d}$ using quantum Monte Carlo (MC) techniques. We find that the system i) is well described by a $1 \mathrm{~d}$ model Hamiltonian with contact interactions and renormalized coupling constant [4] for any value of $a_{3 d}$; ii) reaches the regime of a TG gas for a critical positive value of the $3 \mathrm{~d}$ scattering length $a_{3 d}$; iii) enters a unitary regime for large values of $\left|a_{3 d}\right|$, that is, for $\left|a_{3 d}\right| \rightarrow \infty$, where the properties of the quasi-1d Bose gas become independent of the actual value of $a_{3 d}$ and are similar to those of a 1d gas of hard-rods; and iv) becomes unstable against cluster formation for a critical value of the $1 \mathrm{~d}$ gas parameter, or equivalently, for a critical negative value of the $3 \mathrm{~d}$ scattering length $a_{3 d}$.

The structure of this paper is as follows. Section II discusses the energetics of two bosons in quasi-1d harmonic 
traps. We review the mapping of the $3 \mathrm{~d}$ Hamiltonian to a 1d model Hamiltonian with contact interactions and renormalized coupling constant [4]. The eigenenergies of the system are calculated by exact diagonalization of both the $3 \mathrm{~d}$ and the $1 \mathrm{~d}$ Hamiltonian. We use these results for two particles to benchmark our quantum MC calculations presented in Sec. IV. Section III discusses the relation between the $3 \mathrm{~d}$ and the $1 \mathrm{~d}$ Hamiltonian for $N$ bosons under quasi- $1 \mathrm{~d}$ confinement. Section IV discusses the quantum MC techniques used in the present study: variational, diffusion and fixed-node diffusion MC. The trial wave functions used for the variational estimates and for importance sampling are discussed in detail. Section $\mathrm{V}$ presents our MC results for $N=2$ and $N=10$ atoms in highly-elongated harmonic traps over a wide range of values of the $3 \mathrm{~d}$ scattering length $a_{3 d}$. A comparison of the energetics of the lowest-lying gas-like state for the $3 \mathrm{~d}$ and the $1 \mathrm{~d}$ Hamiltonian is carried out. In the $N=2$ case, we additionally compare with the essentially exact results presented in Sec. II. In the $N=10$ case, we additionally compare with the energy of the lowest-lying gas-like state of the 1d Hamiltonian calculated using the local density approximation (LDA). Section VI discusses the stability of the lowest-lying gas-like state against cluster formation when $a_{3 d}$ is negative using the variational Monte Carlo (VMC) method. We provide a quantitative estimate of the criticality condition. Finally, Sec. VII draws our conclusions.

\section{TWO BOSONS UNDER QUASI-ONE-DIMENSIONAL CONFINEMENT}

Consider two interacting mass $m$ bosons with position vectors $\vec{r}_{1}$ and $\vec{r}_{2}$, where $\vec{r}_{i}=\left(x_{i}, y_{i}, z_{i}\right)$, in a waveguide with harmonic confinement in the radial direction. If we introduce the center of mass coordinate $\vec{R}$ and the relative coordinate $\vec{r}=\vec{r}_{2}-\vec{r}_{1}$, the problem separates. Since the solution to the center of mass Hamiltonian is given readily, we only consider the internal Hamiltonian $H_{3 d}^{i n t}$, which can be conveniently written in terms of cylindrical coordinates $\vec{r}=(\rho, \phi, z)$,

$$
H_{3 d}^{i n t}=-\frac{\hbar^{2}}{2 \mu} \nabla_{\vec{r}}^{2}+V(\vec{r})+\frac{1}{2} \mu \omega_{\rho}^{2} \rho^{2}
$$

where $\mu$ denotes the reduced two-body mass, $\mu=m / 2$, and $V(\vec{r})$ denotes the full $3 \mathrm{~d}$ atom-atom interaction potential.

Considering a regularized zero-range pseudo-potential $V(\vec{r})=2 \pi \hbar^{2} a_{3 d} / \mu \delta(\vec{r}) \frac{\partial}{\partial r} r$, where $a_{3 d}$ denotes the $3 \mathrm{~d}$ scattering length, Olshanii [4] derives an effective $1 \mathrm{~d}$ Hamiltonian,

$$
H_{1 d}^{i n t}=-\frac{\hbar^{2}}{2 \mu} \frac{d^{2}}{d z^{2}}+g_{1 d} \delta(z)+\hbar \omega_{\rho}
$$

and renormalized coupling constant $g_{1 d}$,

$$
g_{1 d}=\frac{2 \hbar^{2} a_{3 d}}{m a_{\rho}^{2}}\left[1-|\zeta(1 / 2)| \frac{a_{3 d}}{\sqrt{2} a_{\rho}}\right]^{-1},
$$

which reproduce the low energy scattering solutions of the full 3d Hamiltonian, Eq. (11). Here, $\zeta(\cdot)$ denotes the Riemann zeta function, $\zeta(1 / 2)=-1.4604$. Alternatively, $g_{1 d}$ can be expressed through the effective $1 \mathrm{~d}$ scattering length $a_{1 d}$ [4],

$$
g_{1 d}=-\frac{2 \hbar^{2}}{m a_{1 d}}
$$

where

$$
a_{1 d}=-a_{\rho}\left(\frac{a_{\rho}}{a_{3 d}}-\frac{|\zeta(1 / 2)|}{\sqrt{2}}\right) .
$$

Olshanii's result shows that the waveguide gives rise to an effective interaction, parameterized by the coupling constant $g_{1 d}$, which can be tuned to any strength by changing the ratio between the $3 \mathrm{~d}$ scattering length $a_{3 d}$ and the transverse oscillator length $a_{\rho}$.

Note that a recent K-matrix treatment [14] results in an explicit energy dependence of the effective $1 \mathrm{~d}$ coupling constant,

$$
\begin{array}{r}
g_{1 d}\left(E_{3 d}^{i n t}\right)=\frac{2 \hbar^{2} a_{3 d}\left(E_{3 d}^{i n t}\right)}{m a_{\rho}^{2}} \times \\
{\left[1-\left|\zeta\left(\frac{1}{2}, \frac{1}{2}\left(3-\frac{E_{3 d}^{i n t}}{\hbar \omega_{\rho}}\right)\right)\right| \frac{a_{3 d}\left(E_{3 d}^{i n t}\right)}{\sqrt{2} a_{\rho}}\right]^{-1}}
\end{array}
$$

where $\zeta(\cdot, \cdot)$ denotes the Hurwitz zeta function and $E_{3 d}^{i n t}$ the internal energy of the two boson system. For scattering between two bosons with minimal internal energy, $E_{3 d}^{i n t}=\hbar \omega_{\rho}$, the Hurwitz zeta function in Eq. (6) reduces to the Riemann zeta function, since $\zeta(0.5,1)=$ $\zeta(0.5)$. With the additional assumption that the energydependence of the $3 \mathrm{~d}$ scattering length $a_{3 d}$ can be neglected, Eq. (6) reduces to Eq. (3). For low-energy scattering the energy-independent effective $1 \mathrm{~d}$ coupling constant, Eq. (3), is expected to provide a good description of the system, and we hence use it throughout this paper.

The renormalized coupling constant, Eq. (3), can be compared with the unrenormalized coupling constant $g_{1 d}^{0}$

$$
g_{1 d}^{0}=\frac{2 \hbar^{2} a_{3 d}}{m a_{\rho}^{2}}
$$

which is obtained by averaging the $3 \mathrm{~d}$ coupling constant $g_{3 d}=4 \pi \hbar^{2} a_{3 d} / m$ over the transverse oscillator ground state (see, e.g., Ref. [5]). Figure [1 shows the unrenormalized coupling constant $g_{1 d}^{0}$ [dashed line, Eq. (7)] together with the renormalized coupling constant [solid line, Eq. (3)]. For $\left|a_{3 d}\right| \ll a_{\rho}$, the renormalized coupling constant $g_{1 d}$ is nearly identical to the unrenormalized coupling constant $g_{1 d}^{0}$. For large $\left|a_{3 d}\right|$, however, the confinement induced renormalization becomes 


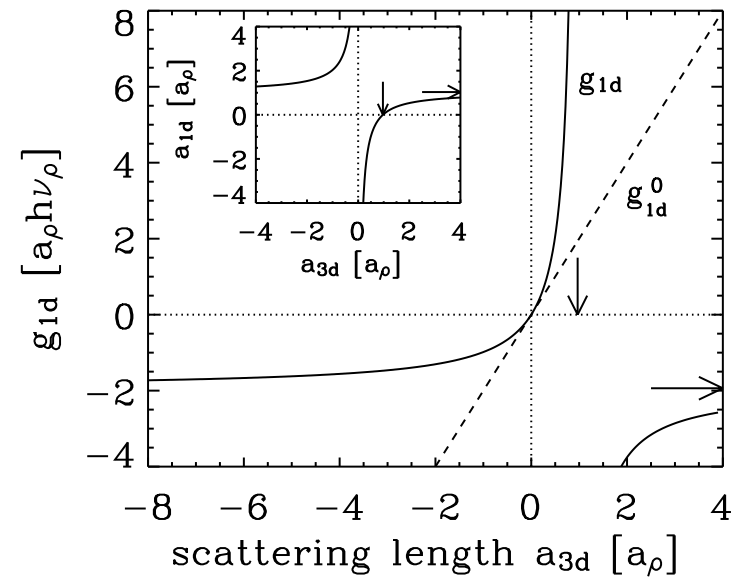

FIG. 1: One-dimensional coupling constants $g_{1 d}$ [Eq. (3]), solid line] and $g_{1 d}^{0}$ [Eq. (7), dashed line] as a function of the $3 \mathrm{~d}$ scattering length $a_{3 d} / a_{\rho}$. The vertical arrow indicates the value of $a_{3 d}$ for which $g_{1 d}$ diverges, $a_{3 d}^{c}=0.9684 a_{\rho}$. The horizontal arrow indicates the asymptotic value of $g_{1 d}$ as $\left|a_{3 d}\right| \rightarrow$ $\infty, g_{1 d}=-1.9368 a_{\rho} \hbar \omega_{\rho}$. Inset: One-dimensional scattering length $a_{1 d}$, Eq. (5), as a function of $a_{3 d} / a_{\rho}$. The vertical arrow indicates the value of $a_{3 d}$ for which $a_{1 d}$ goes through zero, $a_{3 d}^{c}=0.9684 a_{\rho}$. The horizontal arrow indicates the asymptotic value of $a_{1 d}$ as $\left|a_{3 d}\right| \rightarrow \infty, a_{1 d}=1.0326 a_{\rho}$. The angular frequency $\omega_{\rho}$ determines the frequency $\nu_{\rho}, \omega_{\rho}=2 \pi \nu_{\rho}$ (also, $\left.\hbar \omega_{\rho}=h \nu_{\rho}\right)$.

important, and the effective $1 \mathrm{~d}$ coupling constant $g_{1 d}$, Eq. (3), has to be used. At the critical value $a_{3 d}^{c}=$ $0.9684 a_{\rho}$ (indicated by a vertical arrow in Fig. 1), $g_{1 d}$ diverges. For $a_{3 d} \rightarrow \pm \infty, g_{1 d}$ reaches an asymptotic value, $g_{1 d}=-1.9368 a_{\rho} \hbar \omega_{\rho}$ (indicated by a horizontal arrow in Fig. 11). Finally, $g_{1 d}$ is negative for all negative $3 \mathrm{~d}$ scattering lengths. The inset of Fig. 1 shows the effective 1d scattering length $a_{1 d}$, Eq. (5), as a function of $a_{3 d}$. For small positive $a_{3 d}, a_{1 d}$ is negative and it changes sign at $a_{3 d}=a_{3 d}^{c}\left(a_{1 d}=0\right.$ for $\left.a_{3 d}=a_{3 d}^{c}\right)$. Moreover, $a_{1 d}$ reaches, just as $g_{1 d}$, an asymptotic value for $\left|a_{3 d}\right| \rightarrow \infty$, $a_{1 d}=1.0326 a_{\rho}$ (indicated by a horizontal arrow in the inset of Fig. 11). The renormalized 1d scattering length $a_{1 d}$ is positive for negative $a_{3 d}$, and approaches $+\infty$ as $a_{3 d} \rightarrow 0^{-}$. Figure 1 suggests that tuning the $3 \mathrm{~d}$ scattering length $a_{3 d}$ to large values allows a universal quasi-1d regime, where $g_{1 d}$ and $a_{1 d}$ are independent of $a_{3 d}$, to be entered.

The effective coupling constant $g_{1 d}$, Eq. (3), has been derived for a wave guide geometry, that is, with no axial confinement. However, it also describes the scattering between two bosons confined to other quasi-1d geometries. Consider, e.g., a Bose gas under harmonic confinement. If the confinement in the axial direction is weak compared to that of the radial direction, the scattering properties of each atom pair are expected to be described accurately by the effective coupling constant $g_{1 d}$ and the effective scattering length $a_{1 d}$.

The internal motion of two bosons under highlyelongated confinement can be described by the following 3d Hamiltonian

$$
H_{3 d}^{i n t}=-\frac{\hbar^{2}}{2 \mu} \nabla_{\vec{r}}^{2}+V(\vec{r})+\frac{1}{2} \mu\left(\omega_{\rho}^{2} \rho^{2}+\omega_{z}^{2} z^{2}\right),
$$

where $\omega_{z}$ denotes the angular frequency in the longitudinal direction, $\omega_{z}=\lambda \omega_{\rho}$ ( $\lambda$ denotes the aspect ratio, $\lambda \ll 1)$. The eigenenergies $E_{3 d}^{\text {int }}$ and eigenfunctions $\psi_{3 d}^{\text {int }}$ of this Hamiltonian satisfy the Schrödinger equation,

$$
H_{3 d}^{i n t} \psi_{3 d}^{i n t}(\rho, z)=E_{3 d}^{i n t} \psi_{3 d}^{i n t}(\rho, z) .
$$

The corresponding 1d Hamiltonian reads

$$
H_{1 d}^{i n t}=-\frac{\hbar^{2}}{2 \mu} \frac{d^{2}}{d z^{2}}+g_{1 d} \delta(z)+\frac{1}{2} \mu \omega_{z}^{2} z^{2}+\hbar \omega_{\rho} .
$$

The $1 \mathrm{~d}$ eigenenergies $E_{1 d}^{i n t}$ of the time-independent Schrödinger equation,

$$
H_{1 d}^{i n t} \psi_{1 d}^{i n t}(z)=E_{1 d}^{i n t} \psi_{1 d}^{i n t}(z),
$$

can be determined semi-analytically by solving the transcendental equation [15],

$$
g_{1 d}=2 \sqrt{2} \frac{\Gamma\left(\chi_{z}+1\right)}{\Gamma\left(\chi_{z}+1 / 2\right)} \tan \left(\pi \chi_{z}\right) \hbar \omega_{z} a_{z}
$$

self consistently for $\chi_{z}$ (for a given $g_{1 d}$ ). In the above equation, $\chi_{z}$ is an effective (possibly non-integer) quantum number, which determines the energy $E_{z}$,

$$
\chi_{z}=\frac{E_{z}}{2 \hbar \omega_{z}}-\frac{1}{4}
$$

The energy $E_{z}$, in turn, determines the internal $1 \mathrm{~d}$ eigenenergies $E_{1 d}^{\text {int }}$,

$$
E_{1 d}^{i n t}=\lambda E_{z}+\hbar \omega_{\rho} .
$$

In Eq. (12), $a_{z}$ denotes the characteristic oscillator length in the axial direction, $a_{z}=\sqrt{\hbar /\left(m \omega_{z}\right)}$.

To compare the eigenenergies $E_{3 d}^{i n t}$ and $E_{1 d}^{i n t}$, we use, for the $3 \mathrm{~d}$ atom-atom interaction potential $V(r)$, a shortrange (SR) model potential $V^{S R}(r)$ that can support two-body bound states,

$$
V^{S R}(r)=\frac{-V_{0}}{\cosh ^{2}\left(r / r_{0}\right)} .
$$

In the above equation, $V_{0}$ denotes the well depth, and $r_{0}$ the range of the potential. In our calculations, $r_{0}$ is fixed at a value much smaller than the transverse oscillator length, $r_{0}=0.1 a_{\rho}$. To simulate the behavior of $a_{3 d}$ near a field-dependent Feshbach resonance, we vary the well depth $V_{0}$, and consequently, the scattering length $a_{3 d}$. It has been shown that such a model describes many 


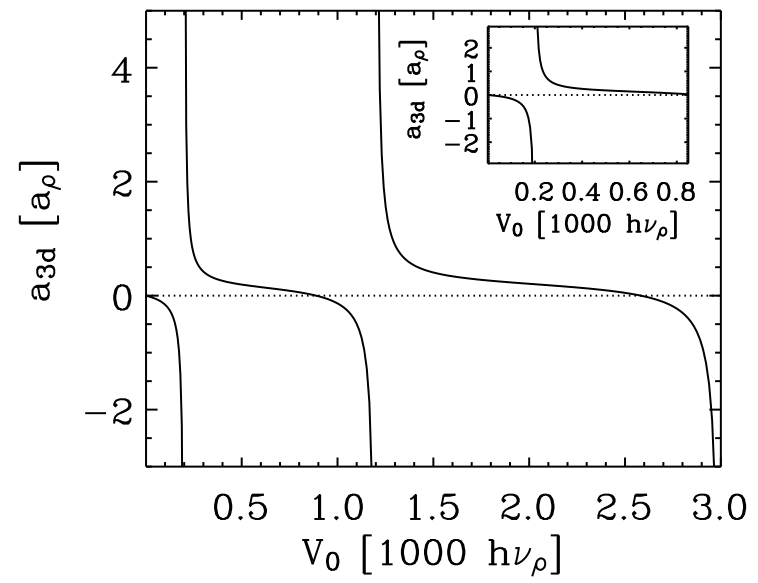

FIG. 2: Three-dimensional $s$-wave scattering length $a_{3 d}$ as a function of the well depth $V_{0}$ for the short-range model potential $V^{S R}$, Eq. (15). Each time the 3d scattering length diverges a new two-body $s$-wave bound state is created. Inset: Enlargement of the well depth region used in our calculations.

atom-atom scattering properties near a Feshbach resonance properly [16]. Figure 2 shows the dependence of the $3 \mathrm{~d}$ scattering length $a_{3 d}$ on $V_{0}$. Importantly, $a_{3 d}$ diverges for particular values of the well depth $V_{0}$. At each of these divergencies, a new two-body $s$-wave bound state is created. The inset of Fig. 2 shows the range of well depths $V_{0}$ used in our calculations.

To benchmark our MC calculations (see Secs. IV and (V), we solve the 3d Schrödinger equation, Eq. (9), with $\lambda=0.01$ for various well depths $V_{0}$ using a twodimensional B-spline basis in $\rho$ and $z$. Figure 3 shows the resulting $3 \mathrm{~d}$ eigenenergies $E_{3 d}^{\text {int }}$ (diamonds) as a function of the $3 \mathrm{~d}$ scattering length $a_{3 d}$. We distinguish between two sets of states: i) States with $E_{3 d}^{i n t} \geq \hbar \omega_{\rho}$ are referred to as gas-like states; their behavior is, to a good approximation, characterized by the $3 \mathrm{~d}$ scattering length $a_{3 d}$, and is hence independent of the detailed shape of the atom-atom potential. The energies of the gas-like states are shown in Fig. 3(a). ii) States with $E_{3 d}^{i n t}<\hbar \omega_{\rho}$ are referred to as molecular-like bound states; their behavior depends on the detailed shape of the atom-atom potential. The energies of these bound states are shown in Fig. 3 b). The well depth $V_{0}$ of the short-range interaction potential $V^{S R}$ is chosen such that $V^{S R}$ supports - in the absence of the confining potential - no $s$-wave bound state for $a_{3 d}<0$, and one $s$-wave bound state for $a_{3 d}>0$. Figure 3(b) shows that the bound state remains bound for $\left|a_{3 d}\right| \rightarrow \infty$ and for negative $a_{3 d}$ if tight radial confinement is present. In addition, a dashed line shows the $3 \mathrm{~d}$ binding energy, $-\hbar^{2} /\left(m a_{3 d}^{2}\right)$, which accurately describes the highest-lying molecular bound state in the absence of any external confinement if $a_{3 d}$ is much larger than the range $r_{0}$ of the potential $V^{S R}$.

The B-spline basis calculations yield not only the in- ternal $3 \mathrm{~d}$ eigenenergies $E_{3 d}^{\text {int }}$, but also the corresponding wave functions $\psi_{3 d}^{\text {int }}$. The nodal surface of the lowestlying gas-like state, which is to a good approximation an ellipse in the $\rho z$-plane, is a crucial ingredient of our many-body calculations. Sections $\mathbb{[ \nabla}$ and $\nabla$ discuss in detail how this nodal surface is used to parametrize our trial wave function entering the $\mathrm{MC}$ calculations.

To compare the energy spectrum for $N=2$ of the effective 1d Hamiltonian with that of the 3d Hamiltonian, Fig. 3 additionally shows the $1 \mathrm{~d}$ eigenenergies $E_{1 d}^{i n t}$ (solid lines) obtained by solving the Schrödinger equation for $H_{1 d}^{\text {int }}$, Eq. (10), semi-analytically [using the renormalized coupling constant $g_{1 d}$, Eq. [3)]. Figure[3(a) demonstrates excellent agreement between the $3 \mathrm{~d}$ and the $1 \mathrm{~d}$ internal energies for all states with gas-like character. For positive $a_{3 d}$, the effective 1d Hamiltonian fails to reproduce the energy spectrum of the molecular-like bound states of the 3d Hamiltonian accurately [see Fig. 33(b), and also Ref. [17, 18]].

Our main focus is in the lowest-lying energy level with gas-like character. This energy branch is shown in Fig. 3(c) on an enlarged scale. A horizontal dashed line shows the lowest internal 3d eigenenergy for two non-interacting spin-polarized fermions (where the antisymmetry of the wave function enters in the $z$ coordinate). Our numerical calculations confirm [17] that for $a_{3 d}=a_{3 d}^{c}\left(g_{1 d} \rightarrow \infty\right)$ the two boson system behaves as if it consisted of two non-interacting spin-polarized fermions (TG gas). The energy $E_{3 d}^{i n t}$ is larger than that of two non-interacting fermions for $a_{3 d}>a_{3 d}^{c}$, and approaches the first excited state energy of two noninteracting bosons for $a_{3 d} \rightarrow 0^{-}$[indicated by a dotted line in Fig. [3(a)].

For positive $g_{1 d}$, the $1 \mathrm{~d}$ Schrödinger equation, Eq. (11), does not support molecular-like bound states. Consequently, the wave function of the lowest-lying gas-like state is positive definite everywhere. For negative $g_{1 d}$, however, one molecular-like two-body bound state exists. If $a_{1 d} \ll a_{z}$ the bound-state wave function is approximately given by the eigenstate $\psi_{1 d}^{\text {int }}$ of the $1 \mathrm{~d}$ Hamiltonian without confinement, Eq. (2),

$$
\psi_{1 d}^{\text {int }}(z)=\exp \left(-\frac{|z|}{a_{1 d}}\right)
$$

with eigenenergy $E_{1 d}^{\text {int }}$,

$$
E_{1 d}^{i n t}=-\frac{\hbar^{2}}{m a_{1 d}^{2}}+\hbar \omega_{\rho}
$$

For the highly-elongated trap with $\lambda=0.01$ shown in Fig. 3(b) and positive $a_{1 d}$ the above binding energy nearly coincides with the exact eigenenergy of the molecular-like bound state obtained from the solution of the transcendental equation (12) (solid line). The twobody binding energy, Eq. (17), is largest for $a_{1 d} \rightarrow 0^{+}$ $\left(g_{1 d} \rightarrow-\infty\right)$; in this case, the molecular-like bound state wave function is tightly-localized around $z=0$, where $z=z_{2}-z_{1}$. Consider a system with $a_{1 d} \ll a_{z}$. For 

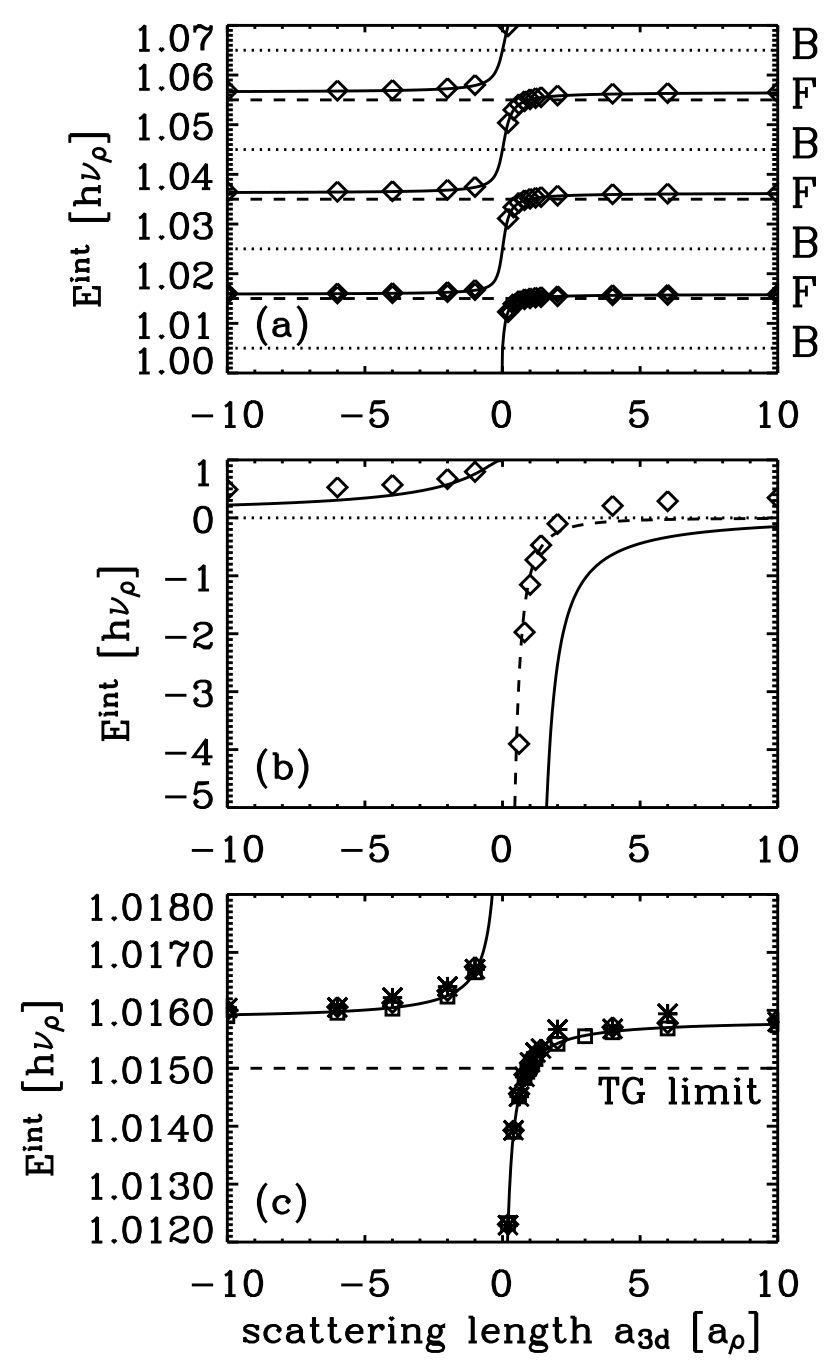

FIG. 3: Internal eigenenergies $E^{\text {int }}$ as a function of the $3 \mathrm{~d}$ scattering length $a_{3 d} / a_{\rho}$ for two bosons under highlyelongated confinement with $\lambda=0.01$. (a) $3 \mathrm{~d} s$-wave eigenenergies $E_{3 d}^{i n t}$ (diamonds) of gas-like states obtained using the short-range model potential $V^{S R}$, Eq. (15), in a B-spline basis set calculation together with internal $1 \mathrm{~d}$ eigenenergies $E_{1 d}^{\text {int }}$ (solid lines). Excellent agreement between the $3 \mathrm{~d}$ and $1 \mathrm{~d}$ energies is found. Horizontal dotted lines show the lowest internal eigenenergies for two non-interacting spin-polarized bosons, while horizontal dashed lines show those for two noninteracting spin-polarized fermions (indicated respectively by "B" and "F" on the right hand side). (b) Binding energy of molecular-like bound states. In addition to the $3 \mathrm{~d}$ and $1 \mathrm{~d}$ energies [diamonds and solid lines, respectively; see (a)], a dashed line shows the $3 \mathrm{~d}$ binding energy $-\hbar^{2} /\left(m a_{3 d}^{2}\right)$. (c) Enlargement of the lowest-lying gas-like state. In addition to the $3 \mathrm{~d}$ and $1 \mathrm{~d}$ energies shown in (a), asterisks show the $3 \mathrm{~d}$ energies for the interaction potential $V^{S R}$ calculated using the FN-DMC technique, and squares the $1 \mathrm{~d}$ energies for the contact interaction potential calculated using the (FN-)DMC technique. The statistical uncertainty of the (FN-)DMC energies is smaller than the symbol size. Good agreement between the (FN-)DMC energies (asterisks and squares) and the energies calculated by alternative means (diamonds and solid lines) is found. negative $g_{1 d}$ (positive $a_{1 d}$ ), the nodes along the relative coordinate $z$ of the lowest-lying gas-like wave function (in this case, the first excited state) are then approximately given by $\pm a_{1 d}$. Thus, imposing the boundary condition $\psi_{1 d}^{\text {int }}=0$ at $|z|=a_{1 d}$ and restricting the configuration space to $z>a_{1 d}$ allows one to obtain an approximation to the eigenenergy of the first excited eigen state. Furthermore, imposing the boundary condition $\psi_{1 d}^{\text {int }}=0$ at $z=a_{1 d}$ is identical to solving the $1 \mathrm{~d}$ Schrödinger equation for a hard-rod interaction potential $V^{H R}(z)$,

$$
V^{H R}(z)=\left\{\begin{array}{ccc}
\infty & \text { for } & z<a_{1 d} \\
0 & \text { for } & z \geq a_{1 d}
\end{array}\right.
$$

For $N=2$, asterisks in Fig. 31(c) show the fixed-node diffusion Monte Carlo (FN-DMC) results obtained using the above fictitious hard-rod potential (see Sec. $\mathrm{VA}$ ). Good agreement is found with the exact $1 \mathrm{~d}$ eigenenergies obtained from Eqs. (12)-(13). For $N>2$ bosons, our 1d FN-DMC algorithm and our usage of the hard-rod equation of state both take advantage of a reduction of configuration space similar to that discussed here for two bosons (see Secs. III and IV).

\section{N BOSONS UNDER QUASI-ONE-DIMENSIONAL CONFINEMENT}

For tightly-confined trapped gases the $1 \mathrm{~d}$ regime is reached if the transverse motion of the atoms is frozen, with all the particles occupying the ground state of the transverse harmonic oscillator. At zero temperature, this condition requires that the energy per particle is dominated by the trapping potential, $E / N=\hbar \omega_{\rho}+\epsilon$, where the excess energy $\epsilon$ is much smaller than the separation between levels in the transverse direction, $\epsilon \ll \hbar \omega_{\rho}$. In the following we consider situations where the Bose gas is in the $1 \mathrm{~d}$ regime for any value of the $3 \mathrm{~d}$ scattering length $a_{3 d}$. For a fixed trap anisotropy parameter $\lambda$ and a fixed number of particles $N$ the above requirement is satisfied if $N \lambda \ll 1$. For $\lambda=0.01$ and $N=10$ (as considered in Sec. $\nabla B$ this condition is fulfilled.

To compare the $3 \mathrm{~d}$ and $1 \mathrm{~d}$ energetics of a Bose gas, we consider the $3 \mathrm{~d}$ and $1 \mathrm{~d}$ Hamiltonian describing $N$ spinpolarized bosons,

$$
\begin{array}{r}
H_{3 d}=\sum_{i=1}^{N}\left[\frac{-\hbar^{2}}{2 m} \nabla_{\vec{r}_{i}}^{2}+\frac{1}{2} m\left(\omega_{\rho}^{2} \rho_{i}^{2}+\omega_{z}^{2} z_{i}^{2}\right)\right]+ \\
\sum_{i<j}^{N} V\left(r_{i j}\right),
\end{array}
$$

and

$$
H_{1 d}=\sum_{i=1}^{N}\left(\frac{-\hbar^{2}}{2 m} \frac{\partial^{2}}{\partial z_{i}^{2}}+\frac{1}{2} m \omega_{z}^{2} z_{i}^{2}\right)+g_{1 d} \sum_{i<j}^{N} \delta\left(z_{i j}\right)+
$$


respectively. The corresponding eigenenergies and eigenfunctions are given by solving the Schrödinger equations,

$$
H_{3 d} \psi_{3 d}\left(\vec{r}_{1}, \cdots, \vec{r}_{N}\right)=E_{3 d} \psi_{3 d}\left(\vec{r}_{1}, \cdots, \vec{r}_{N}\right)
$$

and

$$
H_{1 d} \psi_{1 d}\left(z_{1}, \cdots, z_{N}\right)=E_{1 d} \psi_{1 d}\left(z_{1}, \cdots, z_{N}\right),
$$

respectively. In contrast to Sec. III here we do not separate out the center of mass motion since the MC calculations used to solve the $3 \mathrm{~d}$ and $1 \mathrm{~d}$ many-body Schrödinger equations can be most conveniently implemented in Cartesian coordinate space (see Sec. IV). In the following, we refer to eigenstates of the confined Bose gas with energy greater than $N \hbar \omega_{\rho}$ as gas-like states, and to those with energy smaller than $N \hbar \omega_{\rho}$ as cluster-like bound states.

Section $\nabla B$ compares the energetics of the lowest-lying gas-like state of the 3d Schrödinger equation, Eq. (21), obtained using the short-range potential $\mathrm{V}^{S R}$, Eq. (15), with that obtained using the hard-sphere potential $V^{H S}$,

$$
V^{H S}(r)=\left\{\begin{array}{cc}
\infty & \text { for } \quad r<a_{3 d} \\
0 & \text { for } \quad r \geq a_{3 d}
\end{array}\right.
$$

For $V^{H S}$, the $s$-wave scattering length $a_{3 d}$ coincides with the range of the potential. For $V^{S R}$, in contrast, $r_{0}$ determines the range of the potential, while the scattering length $a_{3 d}$ is determined by $r_{0}$ and $V_{0}$. For $a_{3 d} \ll a_{\rho}$, both potentials give nearly identical results for the energetics of the lowest-lying gas-like state, which depend to a good approximation only on the value of $a_{3 d}$. For $a_{3 d} \gtrsim a_{\rho}$, instead, deviations due to the different effective ranges become visible and only $V^{S R}$ yields results, which do not depend on the short-range details of the potential and which are compatible with a 1d contact potential.

Section $\mathrm{VB}$ also discusses the energetics of the $1 \mathrm{~d}$ Hamiltonian, Eq. (20). For small $\left|g_{1 d}\right|$, the energetics of the many-body 1d Hamiltonian are described well by a 1d mean-field equation with non-linearity. For negative $g_{1 d}$, the mean-field framework describes, for example, bright solitons [20, 21], which have been observed experimentally 22. . For large $\left|g_{1 d}\right|$, in contrast, the system is highly-correlated, and any mean-field treatment will fail. Instead, a many-body description that incorporates higher order correlations has to be used. In particular, the limit $\left|g_{1 d}\right| \rightarrow \infty$ corresponds to the stronglyinteracting $\mathrm{TG}$ regime.

For infinitely strong particle interactions $\left(\left|g_{1 d}\right| \rightarrow \infty\right)$, Girardeau shows [3], using the equivalence between the $1 \mathrm{~d} \delta$-function potential and a "1d hard-point potential", that the energy spectrum of the 1d Bose gas coincides with that of $N$ non-interacting spin-polarized fermions. The lowest eigenenergy per particle of the 1d Bose gas, Eq. (22), is, in the TG limit, given by

$$
\frac{E_{1 d}^{T G}}{N}=\left(\frac{\lambda N}{2}+1\right) \hbar \omega_{\rho}
$$

The corresponding gas density is given by the sum of squares of single-particle wave functions,

$$
n_{1 d}^{T G}(z)=\frac{1}{\sqrt{\pi} a_{z}} \sum_{k=0}^{N-1} \frac{1}{2^{k} k !} H_{k}^{2}\left(z / a_{z}\right) \exp \left[-\left(z / a_{z}\right)^{2}\right]
$$

with the normalization $\int_{-\infty}^{\infty} n_{1 d}^{T G}(z) d z=N$. In Eq. (25), the $H_{k}$ denote Hermite polynomials, and $z$ denotes the distance measured from the center of the trap. For large numbers of atoms, the density expression in Eq. (25) can be calculated using the LDA [23],

$$
n_{1 d}^{T G}(z)=\frac{\sqrt{2 N}}{\pi a_{z}}\left(1-\frac{z^{2}}{2 N a_{z}^{2}}\right)^{1 / 2} .
$$

The above result cannot reproduce the oscillatory behavior of the exact density, Eq. (25), but it does describe the overall behavior properly (see Sec. VII).

To characterize the inhomogeneous 1d Bose gas further, we consider the many-body Hamiltonian of the homogeneous 1d Bose gas,

$$
H_{1 d}^{h o m}=\sum_{i=1}^{N} \frac{-\hbar^{2}}{2 m} \frac{\partial^{2}}{\partial z_{i}^{2}}+g_{1 d} \sum_{i<j}^{N} \delta\left(z_{i j}\right)+N \hbar \omega_{\rho} .
$$

[By introducing the energy shift $N \hbar \omega_{\rho}$, our classification of gas-like states and cluster-like bound states introduced after Eq. (22) remains valid.] For positive $g_{1 d}, H_{1 d}^{h o m}$ corresponds to the Lieb-Liniger (LL) Hamiltonian. The gas-like states of the LL Hamiltonian, including its thermodynamic properties, have been studied in detail [24]. The energy per particle of the lowest-lying gas-like state, the ground state of the system, is given by

$$
\frac{E_{1 d}^{L L}\left(n_{1 d}\right)}{N}=\frac{\hbar^{2}}{2 m} e(\gamma) n_{1 d}^{2}
$$

where $n_{1 d}$ denotes the density of the homogeneous system, and $e(\gamma)$ a function of the dimensionless parameter $\gamma=2 /\left(n_{1 d}\left|a_{1 d}\right|\right)$.

We use the known properties of the LL Hamiltonian to determine properties of the corresponding inhomogeneous system, Eq. (20), within the LDA. This approximation provides a correct description of the trapped gas if the size of the atomic cloud is much larger than the characteristic length scale $a_{z}$ of the confinement in the longitudinal direction 23]. Specifically, consider the local equilibrium condition,

$$
\mu(N)=\hbar \omega_{\rho}+\mu_{\text {local }}\left[n_{1 d}(z)\right]+\frac{1}{2} m \omega_{z}^{2} z^{2},
$$

where $\mu_{\text {local }}\left(n_{1 d}\right)$ denotes the chemical potential of the homogeneous system with density $n_{1 d}$,

$$
\mu_{\text {local }}\left(n_{1 d}\right)=\frac{\partial\left[n_{1 d} E_{1 d}^{L L}\left(n_{1 d}\right) / N\right]}{\partial n_{1 d}} .
$$


The chemical potential $\mu(N)$, Eq. (29), can be calculated using Eq. (30) together with the normalization of the density, $\int_{-\infty}^{\infty} n_{1 d}(z) d z=N$. Integrating the chemical potential $\mu(N)$ then determines the energy of the lowest-lying gas-like state of the inhomogeneous $N$-particle system within the LDA. The LDA treatment is computationally less demanding than solving the many-body Schrödinger equation, Eq. (22), using MC techniques. By comparing with our full $1 \mathrm{~d}$ many-body results we establish the accuracy of the LDA (see Sec. VB).

For negative $g_{1 d}$, the Hamiltonian given in Eq. (27) supports cluster-like bound states ("tightly-bound droplets"). The ground state energy and eigenfunction of the system are [25]

$$
\frac{E_{1 d}^{h o m}}{N}=-\frac{\hbar^{2}}{6 m a_{1 d}^{2}}\left(N^{2}-1\right)+\hbar \omega_{\rho}
$$

and

$$
\psi_{1 d}^{h o m}\left(z_{1}, \cdots, z_{N}\right)=\prod_{i<j}^{N} \exp \left(\frac{-\left|z_{i}-z_{j}\right|}{a_{1 d}}\right)
$$

respectively. The eigenstate given by Eq. (32) depends only on the relative coordinates $z_{i j}$, that is, it is independent of the center of mass of the system. Adding a confinement potential [see Eq. (20)] with $\omega_{z}$ such that $a_{z} \gg a_{1 d}$ leaves the eigenenergy $E_{1 d}^{h o m}$ of this cluster-like bound state to a good approximation unchanged, while the corresponding wave function becomes localized at the center of the trap. This state describes a bright soliton, whose energy can also be determined within a meanfield framework [21]. An excited state of the many-body 1d Hamiltonian with confinement corresponds, e.g., to a state, where $N-1$ particles form a cluster-like bound state, i.e., a soliton with $N-1$ particles, and where one particle approximately occupies the lowest harmonic oscillator state, i.e., has gas-like character. Similarly, molecular-like bound states can form with fewer particles.

The above discussion implies that the lowest-lying gas-like state of the 1d Hamiltonian with confinement, Eq. (20) with negative $g_{1 d}$, corresponds to a highlyexcited state. For dilute 1d systems with negative $g_{1 d}$, the nodal surface of this excited state can be well approximated by the following nodal surface: $\psi_{1 d}=0$ for $z_{i j}=a_{1 d}$, where $i, j=1, \cdots, N$ and $i<j$. As in the twobody case, the many-body energy can then be calculated approximately by restricting the configuration space to regions where the wave function is positive. This corresponds to treating a gas of hard-rods of size $a_{1 d}$. In the low density limit, we expect that the lowest-lying gas-like state of the $1 \mathrm{~d}$ many-body Hamiltonian with $g_{1 d}<0$ is well described by a system of hard-rods of size $a_{1 d}$.

In addition to treating the full $1 \mathrm{~d}$ many-body Hamiltonian, we treat the inhomogeneous system with negative $g_{1 d}$ within the LDA. The equation of state of the uniform hard-rod gas with density $n_{1 d}$ is given by [3]

$$
\frac{E_{1 d}^{H R}\left(n_{1 d}\right)}{N}=\frac{\pi^{2} \hbar^{2} n_{1 d}^{2}}{6 m\left(1-n_{1 d} a_{1 d}\right)^{2}}+\hbar \omega_{\rho} .
$$

We use this energy in the LDA treatment [see Eqs. (28) through (30) with $E_{1 d}^{L L}$ replaced by $\left.E_{1 d}^{H R}\right]$. The hardrod equation of state treated within the LDA provides a good description when $g_{1 d}$ is negative, but $\left|g_{1 d}\right|$ not too small (see Secs. VB and VI). To gain more insight, we determine the expansion for inhomogeneous systems with $N \lambda \ll 1$ using the equation of state for the homogeneous hard-rod gas,

$$
\frac{E_{1 d}}{N}-\hbar \omega_{\rho}=\hbar \omega_{\rho} \frac{N \lambda}{2}\left(1+\frac{128 \sqrt{2}}{45 \pi^{2}} \sqrt{N \lambda} \frac{a_{1 d}}{a_{\rho}}+\cdots\right)
$$

The first term corresponds to the energy per particle in the TG regime [see Eq. 24)]; the other terms can be considered as small corrections to the TG energy. In the unitary limit, that is, for $a_{1 d} / a_{\rho}=1.0326$, expression (34) becomes independent of $a_{3 d}$, and depends only on $N \lambda$. Similarly, the linear density in the center of the cloud, $z=0$, is to lowest order given by the TG result, $n_{1 d}^{T G}(0)=\sqrt{2 N \lambda} /\left(\pi a_{\rho}\right)$ [see Eq. [26)]. Section V1] shows that the TG density provides a good description of inhomogeneous 1d Bose gases over a fairly large range of negative $g_{1 d}$.

\section{QUANTUM MONTE CARLO METHODS}

This section describes the variational, diffusion and fixed-node diffusion MC methods (see, e.g., [26]) used in the present study. These quantum MC techniques solve the many-body Schrödinger equation for the ground state and for excited states at zero temperature. Similar to other MC approaches, these techniques are based on stochastic numerical algorithms, which are powerful when one is treating systems with many degrees of freedom.

\section{A. Variational Monte Carlo method}

The VMC method was first introduced in the seminal work by McMillan 27] to study the ground state properties of liquid ${ }^{4} \mathrm{He}$. The VMC technique is based on the variational principle,

$$
\frac{\left\langle\psi_{T}|H| \psi_{T}\right\rangle}{\left\langle\psi_{T} \mid \psi_{T}\right\rangle} \geq E_{0}
$$

where $\psi_{T}$ denotes a variational or trial wave function, which is parameterized in terms of a set of variational parameters. In Eq. (35), $H$ denotes the Hamiltonian of a bosonic $N$ particle system with ground state energy $E_{0}$ and stationary ground state wave function $\Psi_{0}$. The 
evaluation of the high-dimensional integral, Eq. (35), can be performed by MC techniques, resulting in the $\mathrm{VMC}$ energy expectation value. This variational energy expectation value is an upper bound to the exact ground state energy $E_{0}$. Importantly, the variational principle also applies to excited states. For a trial wave function $\psi_{T}$ with a given symmetry, the variational estimate provides an upper bound to the energy of the lowest excited state of the Hamiltonian $H$ with that symmetry.

Choosing a good functional form for the trial wave function $\psi_{T}$ is a crucial step of the VMC method (and also of the DMC method and the FN-DMC method, see Secs. IVB and IVC). A general ansatz for $\psi_{T}$, which has been used successfully to describe a system of $N$ spinless bosons under external confinement in either $3 \mathrm{~d}$ or $1 \mathrm{~d}$ [28], is a Bijl-Jastrow decomposition into one- and two-body correlation factors. The one-body term accounts for the confining potential, while the two-body term accounts for interactions between particles.

To describe the lowest-lying gas-like state of $H_{3 d}$ [Eq. [19] ], we use the following trial wave function

$$
\psi_{T}^{3 d}\left(\vec{r}_{1}, \ldots, \vec{r}_{N}\right)=\prod_{i=1}^{N} e^{-z_{i}^{2} /\left(2 \alpha_{z}^{2}\right)} e^{-\rho_{i}^{2} /\left(2 \alpha_{\rho}^{2}\right)} \prod_{i<j}^{N} f_{2}\left(\vec{r}_{i}-\vec{r}_{j}\right) .
$$

Here, $\alpha_{z}$ and $\alpha_{\rho}$ determine the Gaussian width of $\psi_{T}^{3 d}$ in the longitudinal and transverse direction, respectively. These variational parameters are optimized in the course of the VMC calculation by minimizing the energy expectation value. The two-body correlation factor $f_{2}(\vec{r})$ is chosen to closely reproduce the scattering behavior of two bosons at low energies. For the hard-sphere potential, we use

$$
f_{2}(\vec{r})=\left\{\begin{array}{ccc}
0 & \text { for } & |\vec{r}| \leq a_{3 d} \\
1-a_{3 d} /|\vec{r}| & \text { for } & |\vec{r}|>a_{3 d}
\end{array}\right.
$$

The constraint $f_{2}=0$ for $r \leq a_{3 d}$ accounts for the boundary condition imposed by the hard-sphere potential; it is exact even for the many-body system. For the shortrange potential, we use instead

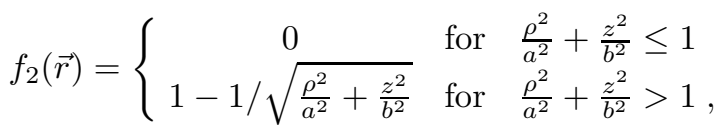

where $a$ and $b$ denote the lengths of the semi-axes of an ellipse. For two particles under highly-elongated confinement, the nodal surface is to a good approximation ellipticly shaped (see Sec. $\sqrt{ }$ ). Thus, the parameters $a$ and $b$ are determined by fitting the elliptical surface to the nodal surface obtained by solving the Schrödinger equation for $N=2$, Eqs. (8) and (9), by performing a B-spline basis set calculation. In contrast to $V^{H S}$, the constraint $f_{2}=0$ in Eq. (38) parameterizes the manybody nodal surface for $V^{S R}$ only approximately. We expect that our parameterization leads to an accurate description of quasi-1d Bose gases if the average distance between particles is much larger than the semi-axes of the ellipse. The trial wave functions discussed here in the context of our VMC calculations also enter our (FN)DMC calculations (see Sec. IVB and IV C).

In the case of the 1d Hamiltonian, Eq. (20), we use a trial wave function of the form

$$
\psi_{T}^{1 d}\left(z_{1}, \ldots, z_{N}\right)=\prod_{i=1}^{N} e^{-z_{i}^{2} /\left(2 \alpha_{z}^{2}\right)} \prod_{i<j}^{N} f_{2}\left(z_{i}-z_{j}\right),
$$

where the Gaussian width $\alpha_{z}$ is treated as a variational parameter. The two-body correlation factor $f_{2}(z)$ is chosen as

$$
f_{2}(z)=\left\{\begin{array}{ccc}
\cos \left[k_{z}(|z|-\bar{Z})\right] & \text { for } & |z| \leq \bar{Z} \\
1 & \text { for } & |z|>\bar{Z}
\end{array}\right.
$$

The cut-off length $\bar{Z}$ is fixed at $\bar{Z}=500 a_{1 d}$, while the wave vector $k_{z}$ is chosen such that the boundary condition at $z=0$ imposed by the $\delta$-function potential is satisfied: $-k_{z} \tan \left(k_{z} \bar{Z}\right)=1 / a_{1 d}$. For negative $a_{1 d}\left(g_{1 d}>0\right)$ the correlation function, Eq. (40), is positive everywhere. For positive $a_{1 d}\left(g_{1 d}<0\right)$, in contrast, $f_{2}(z)$ changes sign at $|z|=a_{1 d}$. The parameterization given by Eq. (40) is used in our stability analysis performed within a VMC framework (see Sec. VI) and in our DMC calculations for $g_{1 d}>0$ (see Sec. IVB). To perform the FN-DMC calculations for negative $g_{1 d}$ (see Sec. IV C), we need to construct a trial wave function that is positive definite everywhere. In the FN-DMC calculations, we thus use an alternative parameterization, which imposes the constraint $f_{2}=0$ for $a_{1 d} \leq z$,

$$
f_{2}(z)=\left\{\begin{array}{cl}
0 & \text { for } z \leq a_{1 d} \\
\cos \left[k_{z}(|z|-\bar{Z})\right] & \text { for } a_{1 d}<z \leq \bar{Z} \\
1 & \text { for } z>\bar{Z}
\end{array}\right.
$$

\section{B. Diffusion Monte Carlo method}

The diffusion Monte Carlo (DMC) algorithm solves the time-independent Schrödinger equation of a $N$ particle system by introducing the imaginary time $\tau=i t / \hbar$, and propagating the function $f(\mathcal{R}, \tau)=\psi_{T}(\mathcal{R}) \Psi(\mathcal{R}, \tau)$ in imaginary time $\tau$. Here, $\Psi(\mathcal{R}, \tau)$ denotes the wave function of the system, which we are seeking. The trial wave function $\psi_{T}(\mathcal{R})$, which can be optimized in a series of VMC calculations, enters the DMC calculations as input. In $3 \mathrm{~d}, \mathcal{R}$ collectively denotes the position vectors, $\mathcal{R}=\left(\vec{r}_{1}, \ldots, \vec{r}_{N}\right)$; in $1 \mathrm{~d}$, it collectively denotes the positions, $\mathcal{R}=\left(z_{1}, \cdots, z_{N}\right)$.

Using the function $f(\mathcal{R}, \tau)$, the time-dependent Schrödinger equation in imaginary time can be rewritten as

$$
\begin{aligned}
-\frac{\partial f(\mathcal{R}, \tau)}{\partial \tau}= & -D \nabla_{\mathcal{R}}^{2} f(\mathcal{R}, \tau)+D \nabla_{\mathcal{R}}[F(\mathcal{R}) f(\mathcal{R}, \tau)] \\
& +\left[E_{L}(\mathcal{R})-E_{\text {ref }}\right] f(\mathcal{R}, \tau)
\end{aligned}
$$

where $E_{L}(\mathcal{R})=\psi_{T}(\mathcal{R})^{-1} H \psi_{T}(\mathcal{R})$ denotes the local energy, $F(\mathcal{R})=2 \psi_{T}(\mathcal{R})^{-1} \nabla_{\mathcal{R}} \psi_{T}(\mathcal{R})$ the quantum drift 
force, and $D$ the diffusion constant, $D=\hbar^{2} / 2 m$. The subscript $\mathcal{R}$ of the operator $\nabla$ indicates that the derivative has to be taken for every component of $\mathcal{R}$. The constant energy shift $E_{r e f}$ is introduced to stabilize the numerics. The solution of Eq. (42) can be written formally as

$$
f\left(\mathcal{R}^{\prime}, \tau+\Delta \tau\right)=\int d \mathcal{R} G\left(\mathcal{R}^{\prime}, \mathcal{R}, \Delta \tau\right) f(\mathcal{R}, \tau),
$$

where the time-dependent Green's function $G$ is given by

$$
G\left(\mathcal{R}^{\prime}, \mathcal{R}, \Delta \tau\right)=\left\langle\mathcal{R}^{\prime}\left|e^{-A \Delta \tau}\right| \mathcal{R}\right\rangle .
$$

Here, $A$ denotes the time evolution operator of Eq. (42), $-\partial f(\mathcal{R}, \tau) / \partial \tau=A f(\mathcal{R}, \tau)$. If the short-time approximation to the Green's function $G\left(\mathcal{R}^{\prime}, \mathcal{R}, \Delta \tau\right)$ is known (for sufficiently small $\Delta \tau$ ), the asymptotic solution for large times $\tau, f(\mathcal{R}, \tau \rightarrow \infty)$, can be obtained by propagating $f$ for a large number of time steps $\Delta \tau$ (for more details see, e.g., [29]). For a system of bosons, one can show that $f(\mathcal{R}, \tau \rightarrow \infty)=\psi_{T}(\mathcal{R}) \Psi_{0}(\mathcal{R})$, where $\Psi_{0}$ is the exact ground-state eigenfunction. We calculate the eigenenergy $E_{0}$ by first propagating to large times $\tau$, and then averaging the local energy $E_{L}$ over the distribution $f(\mathcal{R}, \tau)$,

$E_{0}=\frac{\int d \mathcal{R} \psi_{T}(\mathcal{R}) H \Psi_{0}(\mathcal{R})}{\int d \mathcal{R} \psi_{T}(\mathcal{R}) \Psi_{0}(\mathcal{R})}=\frac{\int d \mathcal{R} f(\mathcal{R}, \tau \rightarrow \infty) E_{L}(\mathcal{R})}{\int d \mathcal{R} f(\mathcal{R}, \tau \rightarrow \infty)}$

Apart from statistical errors, the DMC method determines the energy of the nodeless ground state of a system of $N$ bosons essentially exactly. Importantly, the energy expectation value calculated by the DMC technique with importance sampling, which we use in our study, is independent of the detailed shape of $\psi_{T}$ as long as $\psi_{T}$ is positive definite everywhere.

We use the outlined algorithm to calculate the groundstate energy $E_{3 d}$ of the 3d Hamiltonian, Eq. (19), for the hard-sphere interaction potential, $V^{H S}$, and that of the 1d Hamiltonian, Eq. (20), for positive coupling constant, $g_{1 d}>0$. The trial wave function $\psi_{T}$ used in these cases is given by Eqs. (36) and (37), and by Eqs. (39) and (40), respectively.

The DMC algorithm cannot be used directly to calculate excited states. If $\Psi$ is an excited state, that is, if $\Psi$ is orthogonal to $\Psi_{0}$, the function $f(\mathcal{R}, \tau)=\psi_{T}(\mathcal{R}) \Psi(\mathcal{R}, \tau)$ is not positive everywhere in configuration space and can hence not be interpreted as a probability density. This leads to the so-called sign problem. To nevertheless calculate excited state energies such as the energy of the lowest-lying gas-like state of the 3d Hamiltonian, Eq. (19), with the short-range interatomic potential $V^{S R}$, Eq. (15), or of the 1d Hamiltonian, Eq. (20) with $g_{1 d}<0$, we apply the FN-DMC method.

\section{Fixed-node diffusion Monte Carlo method}

The FN-DMC method [30] modifies the DMC method to allow approximate treatment of excited states of many-body systems. The idea of the FN-DMC method is to treat excited states by "enforcing" the positive definiteness of the probability distribution $f(\mathcal{R}, \tau)=$ $\psi_{T}(\mathcal{R}) \Psi(\mathcal{R}, \tau)$. The function $f$ is positive definite everywhere in configuration space, and can hence be interpreted as a probability distribution, if $\psi_{T}$ and $\Psi$ change sign together, and thus share the same (highdimensional) nodal surface. To ensure positive definiteness of $f$, the trial wave function $\psi_{T}$ imposes a nodal constraint, which is fixed during the calculation. Within this constraint, the function $f$ is propagated (following a scheme very similar to that outlined in Sec. IVB), and reaches an asymptotic distribution for large $\tau, f(\mathcal{R}, \tau \rightarrow$ $\infty)=\psi_{T}(\mathcal{R}) \Psi(\mathcal{R})$. In the FN-DMC method, $\Psi$ is an approximation to the exact excited eigenfunction of the many-body Schrödinger equation (and not the exact eigenfunction as in the DMC method). It can be proven that, due to the nodal constraint, the fixed-node energy is a variational upper bound to the exact eigenenergy for a given symmetry [30]. In particular, if the nodal surface of $\psi_{T}$ were exact, then $\Psi$ would be exact. Thus, the FN-DMC energy depends crucially on a good parameterization of the many-body nodal surface.

Section $\nabla$ reports the energy of the lowest-lying gaslike state of the 3d Hamiltonian, Eq. (19), for the shortrange potential $V^{S R}$, and that of the 1d Hamiltonian, Eq. (20), for $g_{1 d}<0$ calculated using the FN-DMC method. As discussed in Sec. IVA the nodal surface of the many-body trial wave function $\psi_{T}$ is constructed from the essentially exact nodal surface of the two-body wave function describing the lowest-lying gas-like state. In the $3 \mathrm{~d}$ case, the importance sampling trial wave function is given by Eqs. (36) and (38). In the $1 \mathrm{~d}$ case, the importance sampling trial wave function is instead given by Eqs. (39) and (41). We expect that the FN-DMC approach implemented here results in accurate many-body energies of dilute quasi-1d Bose gases.

\section{ENERGETICS OF QUASI-ONE-DIMENSIONAL BOSE GASES}

Table【summarizes the techniques used to solve the $1 \mathrm{~d}$ and 3d Hamiltonian, respectively. This table is meant to guide the reader through our result sections. Section $\nabla \mathrm{A}$ discusses our MC energies for two-particle systems, while Sec. $\mathrm{VB}$ discusses the energetics for larger systems, calculated within various frameworks. Finally, Sec. VI discusses the stability of quasi-1d Bose gases.

\section{A. Two-body system}

Section [iscusses the calculation of the energy spectrum related to the internal motion of two bosons under highly-elongated confinement, Eq. (8), using a B-spline basis, and the eigenspectrum related to the internal motion of two bosons under 1d confinement, Eq. (10), using 


\begin{tabular}{l|l|l|l} 
Hamiltonian & interaction & technique & Section \\
\hline$H_{3 d}$ & $V^{H S}$ & DMC & VB \\
$H_{3 d}$ & $V^{S R}$ & FN-DMC & VA, VB \\
\hline$H_{1 d}$ & $g_{1 d}>0$ & DMC & VA, VB \\
$H_{1 d}$ & $g_{1 d}>0$ & LDA, LL & VB \\
$H_{1 d}$ & $g_{1 d}<0$ & FN-DMC & VA, VB \\
$H_{1 d}$ & $g_{1 d}>0$ & LDA, hard-rod & VB \\
$H_{1 d}$ & $g_{1 d}<0$ & VMC & VI
\end{tabular}

TABLE I: Guide that summarizes the techniques used to solve the $3 \mathrm{~d}$ and $1 \mathrm{~d}$ Hamiltonian, Eqs. (19) and (20), respectively. Column 2 specifies the atom-atom interactions of the many-body Hamiltonian, column 3 lists the techniques used to solve the corresponding many-body Schrödinger equation, and column 4 lists the sections that discuss the results obtained using this approach.

Eqs. (12) through (14). We now use these essentially exact eigenenergies to benchmark our (FN-)DMC calculations. Toward this end, we solve the $3 \mathrm{~d}$ Schrödinger equation, Eq. (19), and the 1d Schrödinger equation, Eq. (20), for various interaction strengths for $N=2$ and $\lambda=0.01$ using (FN-)DMC techniques. The resulting MC energies $E_{3 d}$ and $E_{1 d}$ include the center of mass energy of $(1+\lambda / 2) \hbar \omega_{\rho}$. To compare with the internal eigenenergies discussed in Sec. III we subtract the center of mass energy from the (FN-)DMC energies.

For $N=2$, the lowest-lying gas-like state of the $3 \mathrm{~d}$ Hamiltonian for the short-range potential $V^{S R}$ is the first excited eigenstate. Consequently, we solve the $3 \mathrm{~d}$ Schrödinger equation by the FN-DMC technique using the trial wave function $\psi_{T}$ given by Eqs. (36) and (38). Figure 4 shows the elliptical nodal surface of the trial wave function $\psi_{T}$ (solid lines) together with the essentially exact nodal surface calculated using a B-spline basis set (symbols; see also Sec. III) for $\lambda=0.01$ and three different scattering lengths, $a_{3 d} / a_{\rho}=1,6$, and -4 . Notably, the semi-axes $a$ along the $\rho$ coordinate is larger than that along the $z$ coordinate $(a / b>1)$, "opposing" the shape of the confining potential, for which the characteristic length along the $\rho$ coordinate is smaller than that along the $z$ coordinate $\left(a_{\rho} / a_{z}<1\right)$. Figure 4 indicates good agreement between the essentially exact nodal surface and the parameterization of the nodal surface by an ellipse for $a_{3 d} / a_{\rho}=1$ and 6 ; some discrepancies become apparent for negative $a_{3 d}$. Since the FN-DMC method results in the exact energy if the nodal surface of $\psi_{T}$ coincides with the nodal surface of the exact eigenfunction, comparing the FN-DMC energies for two particles with those obtained from a B-spline basis set calculation provides a direct measure of the quality of the nodal surface of $\psi_{T}$. Figure 3(c) compares the internal 3d energy of the lowest-lying gas-like state calculated using a Bspline basis (diamonds, see Sec. III) with that calculated using the FN-DMC technique (asterisks). The agreement between these two sets of energies is - within the statistical uncertainty - excellent for all scattering lengths

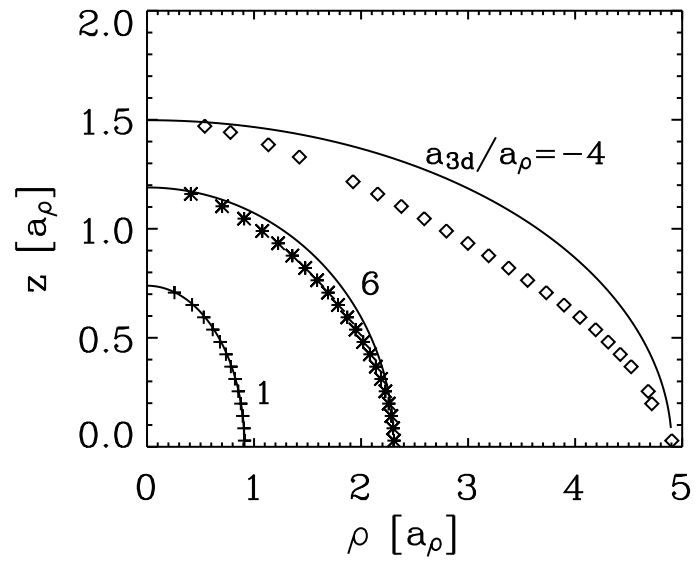

FIG. 4: Nodal surface of the trial wave function $\psi_{T}$ [solid lines, Eq. [38)] together with the essentially exact nodal surface calculated using a B-spline basis set (symbols) for $\lambda=0.01, N=2$, and three different scattering lengths, $a_{3 d} / a_{\rho}=1$ (pluses), $a_{3 d} / a_{\rho}=6$ (asterisks), and $a_{3 d} / a_{\rho}=-4$ (diamonds). The nodal surface is shown as a function of the internal coordinates $z$ and $\rho$. Good agreement between the elliptical nodal surface (solid lines) and the essentially exact nodal surface (symbols) is visible for $a_{3 d} / a_{\rho}=1$ and 6 . Small deviations are visible for $a_{3 d} / a_{\rho}=-4$.

$a_{3 d}$ considered. We conclude that our parameterization of the two-body nodal surface, Eq. (38), is accurate over the whole range of interaction strengths $a_{3 d}$ considered.

We expect that our parameterization of the two-body nodal surface is to a good approximation independent of the confining potential in $z$ (for small enough $\lambda$ ). In fact, we expect our nodal surface to closely resemble that of the scattering wave function at low scattering energy of the $3 \mathrm{~d}$ wave guide Hamiltonian given by Eq. (11). To quantify this statement, Fig. [5]shows the semi-axes $a$ and $b$ (pluses and asterisks, respectively) obtained by fitting an ellipse, see Eq. (38), to the nodal surface obtained by solving the Schrödinger equation for the two-body Hamiltonian, Eq. (8), using a B-spline basis for various aspect ratios $\lambda(\lambda=0.001, \cdots, 1)$, and fixed scattering length, $a_{3 d}=2 a_{\rho}$ (similar results are found for other scattering lengths). Indeed, the nodal surface for a given $a_{3 d} / a_{\rho}$ is nearly independent of the aspect ratio $\lambda$ for $\lambda \leq 0.01$. These findings for two particles imply that the parameterization of the nodal surface of $\psi_{T}$ used in the FNDMC many-body calculations should be good as long as the density along $z$ is small.

Next, consider the 1d Hamiltonian, Eq. (20), for $N=$ 2. For positive $g_{1 d}$, the lowest-lying gas-like state is the ground state of the two-body system and we hence use the DMC technique [with $\psi_{T}$ given by Eqs. (39) and [40]]; for $g_{1 d}<0$, the lowest-lying gas-like state is the first excited state, and we instead use the FN-DMC technique [with $\psi_{T}$ given by Eqs. (39) and (41)]. Figure 3 


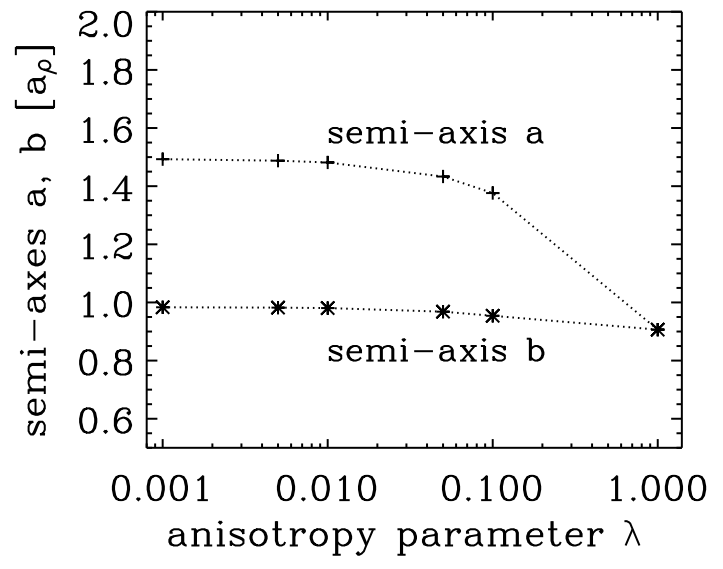

FIG. 5: Semi-axes $a$ (pluses) and $b$ (asterisks) obtained by fitting an ellipse [see Eq. (38)] to the essentially exact nodal surface for two bosons under cylindrical confinement, calculated using a B-spline basis set as a function of the anisotropy parameter $\lambda$, for $a_{3 d} / a_{\rho}=2$. Dotted lines are shown to guide the eye. For $\lambda \leq 0.01$, the nodal surface is nearly independent of the anisotropy parameter $\lambda$.

shows the 1d energies of the lowest-lying gas-like state calculated using Eqs. (12) through (14) (solid line), together with those calculated by the (FN-)DMC technique (squares). We find excellent agreement between these two sets of $1 \mathrm{~d}$ energies.

The comparison for two bosons between the (FN-)$\mathrm{DMC}$ energies and the energies calculated by alternative means serves as a stringent test of our MC codes, since these codes are implemented such that the number of particles enters simply as a parameter.

\section{B. N-body system}

This section presents our many-body study, which investigates the properties of quasi-1d Bose gases over a wide range of scattering lengths $a_{3 d}$. We focus specifically on three distinct regimes: i) $0<a_{3 d}<a_{3 d}^{c}$ ( $g_{1 d}$ is positive); ii) $\left|a_{3 d}\right| \rightarrow \infty$ ( $g_{1 d}$ and $a_{1 d}$ are independent of $a_{3 d}$; unitary regime); and iii) $a_{3 d} \rightarrow 0^{-}$( $a_{1 d}$ is large and positive; onset of instability). We discuss the energetics of quasi-1d Bose gases for $N=10$. Our results presented here support our earlier conclusions, which are based on a study conducted for a smaller system, i.e., for $N=5 \underline{13}$.

For small $\lambda$ (here, $\lambda=0.01$ ), the radial angular frequency $\omega_{\rho}$ dominates the eigenenergies of the $3 \mathrm{~d}$ and of the 1d Schrödinger equation. The shift of the eigenenergy of the lowest-lying gas-like state as a function of the interaction strength is, however, set by the axial angular frequency $\omega_{z}$. To emphasize the dependence of the eigenenergies of the lowest-lying gas-like state on $\omega_{z}$, we

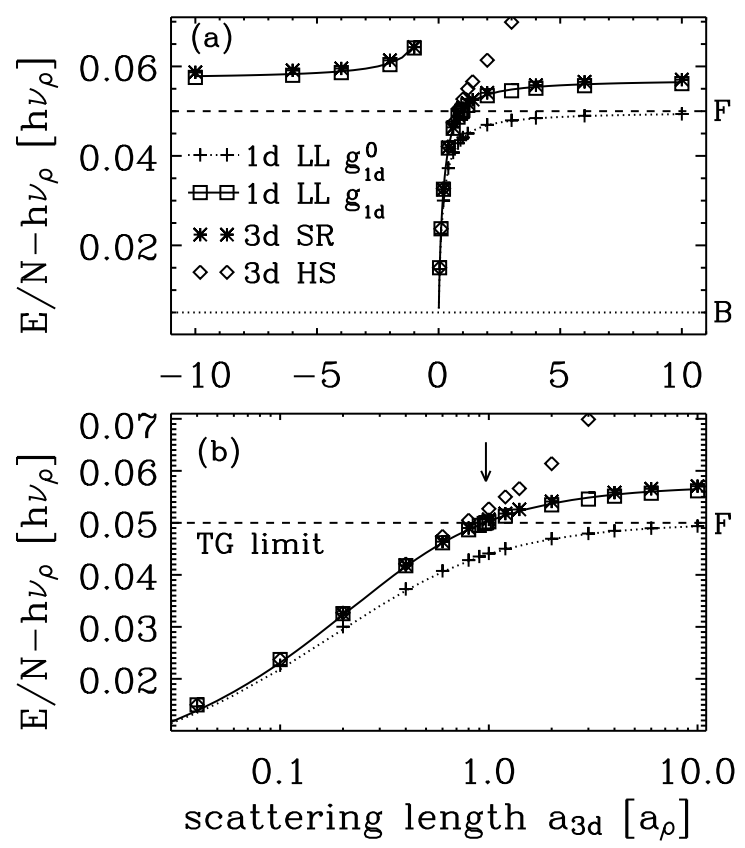

FIG. 6: Three-dimensional (FN-)DMC energy per particle, $E_{3 d} / N-\hbar \omega_{\rho}$, calculated using $V^{H S}$ (diamonds) and $V^{S R}$ (asterisks), respectively, together with 1d (FN-)DMC energy per particle, $E_{1 d} / N-\hbar \omega_{\rho}$, calculated using $g_{1 d}$ [squares, Eq. (3)] and $g_{1 d}^{0}$ [pluses, Eq. (7)], respectively, as a function of $a_{3 d}$ [(a) linear scale; (b) logarithmic scale] for $N=10$ and $\lambda=0.01$. The statistical uncertainty of the (FN-)DMC energies is smaller than the symbol size. Dotted and solid lines show the 1d energy per particle calculated within the LDA for $g_{1 d}^{0}$, Eq. (7) [using the LL equation of state] and for $g_{1 d}$, Eq. (3) [using the LL equation of state for $g_{1 d}>0$, and the hard-rod equation of state for $\left.g_{1 d}<0\right]$, respectively. A dotted horizontal line indicates the energy per particle of a noninteracting Bose gas, and a dashed horizontal line indicates the TG energy per particle. A vertical arrow the position where $g_{1 d}$, Eq. (3), diverges.

report the energy per particle subtracting the constant offset $\hbar \omega_{\rho}$, that is, we report the quantity $E / N-\hbar \omega_{\rho}$.

Consider the lowest-lying gas-like state of the $3 \mathrm{~d}$ Schrödinger equation. Figure [6 shows the 3d energy per particle, $E_{3 d} / N-\hbar \omega_{\rho}$, as a function of $a_{3 d}$ for $N=10$ under quasi-1d confinement, $\lambda=0.01$, for the hard-sphere two-body potential $V^{H S}$ (diamonds) and the short-range potential $V^{S R}$ (asterisks). The energies for $V^{H S}$ are calculated using the DMC method [with $\psi_{T}$ given by Eqs. (36) and (37)], while those for $V^{S R}$ are calculated using the FN-DMC method [with $\psi_{T}$ given by Eqs. (36) and (38)]. For small $a_{3 d} / a_{\rho}$, the energies for these two two-body potentials agree within the statistical uncertainty. For $a_{3 d} \gtrsim a_{\rho}$, however, clear discrepancies are visible. The DMC energies for $V^{S R}$ cross the TG energy per particle (indicated by a dashed horizontal line), $E / N-\hbar \omega_{\rho}=\hbar \omega_{\rho} \lambda N / 2$, very close to the value $a_{3 d}^{c}=0.9684 a_{\rho}$ [indicated by a vertical arrow in Fig. [6(b)], while the energies for $V^{H S}$ cross the TG en- 
ergy per particle at a somewhat smaller value of $a_{3 d}$. For $V^{H S}$, "fermionization" of a quasi-1d gas has previously been investigated in detail [19]. The present paper goes beyond these previous studies in that we consider a short-range potential, whose applicability extends to the regime $a_{3 d}>a_{3 d}^{c}$.

For $a_{3 d}>a_{3 d}^{c}$, the energy for the short-range potential $V^{S R}$ of the lowest-lying gas-like state increases slowly with increasing $a_{3 d}$, and becomes approximately constant for large values of $\left|a_{3 d}\right|$. The limit $\left|a_{3 d}\right| \rightarrow \infty$ corresponds to the unitary regime (see below). Notably, the $3 \mathrm{~d}$ energy behaves smoothly as $a_{3 d}$ diverges. The $3 \mathrm{~d}$ energy slowly increases further for increasing negative $a_{3 d}$, and changes more rapidly as $a_{3 d} \rightarrow 0^{-}$. The $\left|a_{3 d}\right| \rightarrow \infty$ regime and the $a_{3 d} \rightarrow 0^{-}$regime are discussed in more detail below.

To compare our results obtained for the 3d Hamiltonian, $H_{3 d}$, with those for the $1 d$ Hamiltonian, $H_{1 d}$, we also solve the Schrödinger equation for $H_{1 d}$, Eq. (20), for the lowest-lying gas-like state. For positive coupling constants, $g_{1 d}>0$, the lowest-lying gas-like state is the many-body ground state, and we hence use the DMC method [with $\psi_{T}$ given by Eqs. (39) and (40)]. For $g_{1 d}<0$, however, the 1d Hamiltonian supports clusterlike bound states. In this case, the lowest-lying gas-like state corresponds to an excited many-body state, and we hence solve the 1d Schrödinger equation by the FN-DMC method [with $\psi_{T}$ given by Eqs. (39) and (41)].

Figure 6] shows the resulting 1d energies per particle, $E_{1 d} / N-\hbar \omega_{\rho}$, for the renormalized coupling constant $g_{1 d}$ [squares, Eq. (3)], and the unrenormalized coupling constant $g_{1 d}^{0}$ [pluses, Eq. (7)], respectively. The 1d energies calculated using the two different coupling constants agree well for small $a_{3 d}$, while clear discrepancies become apparent for $a_{3 d} \gtrsim a_{3 d}^{c}$. In fact, the $1 d$ energies calculated using the unrenormalized coupling constant $g_{1 d}^{0}$ approach the TG energy (dashed horizontal line) asymptotically for $a_{3 d} \rightarrow \infty$, but do not become larger than the TG energy. The 1d energies calculated using the renormalized $1 \mathrm{~d}$ coupling constant $g_{1 d}$ agree well with the $3 \mathrm{~d}$ energies calculated using the short-range potential $V^{S R}$ (asterisks) up to very large values of the $3 \mathrm{~d}$ scattering length $a_{3 d}$. In contrast, the $1 \mathrm{~d}$ energies deviate clearly from the $3 \mathrm{~d}$ energies calculated using the hard-sphere potential $V^{H S}$ (diamonds) at large $a_{3 d}$.

The $1 \mathrm{~d}$ energies calculated using the renormalized coupling constant agree with the $3 \mathrm{~d}$ energies calculated using the short-range potential $V^{S R}$ also for $\left|a_{3 d}\right| \rightarrow \infty$, that is, in the unitary regime, and for negative $a_{3 d}$. Small deviations between the $1 \mathrm{~d}$ energies calculated using the renormalized $1 \mathrm{~d}$ coupling constant $g_{1 d}$ and the $3 \mathrm{~d}$ energies calculated using the short-range potential $V^{S R}$ are visible; we attribute these to the finite range of $V^{S R}$. The deviations should decrease with decreasing range $r_{0}$ of the short-range potential $V^{S R}$. On the other hand, $r_{0}$ determines to first order the energy-dependence of the scattering length $a_{3 d}$. Thus, usage of an energy-dependent coupling constant $g_{1 d}$ [see Eq. [6] should also reduce the deviations between the $1 \mathrm{~d}$ energies and the $3 \mathrm{~d}$ energies calculated using the short-range potential $V^{S R}$ [14]. Such an approach is, however, beyond the scope of this paper.

We conclude that the renormalization of the effective $1 \mathrm{~d}$ coupling constant $g_{1 d}$ and of the $1 \mathrm{~d}$ scattering length $a_{1 d}$ are crucial to reproduce the results of the $3 \mathrm{~d}$ Hamiltonian $H_{3 d}$ when $a_{3 d} \gtrsim a_{\rho}$ and when $a_{3 d}$ is negative.

In addition to treating the $1 \mathrm{~d}$ many-body Hamiltonian using the (FN-)DMC technique, we solve the $1 \mathrm{~d}$ Schrödinger equation using the LL equation of state $\left(g_{1 d}>0\right)$ and the hard-rod equation of state $\left(g_{1 d}<0\right)$ within the LDA (see Sec. III). These treatments are expected to be good when the size of the cloud is much larger than the harmonic oscillator length $a_{z}$, where $a_{z}=\sqrt{\hbar / m \omega_{z}}$, that is, when $a_{3 d}$ is large and positive or when $a_{3 d}$ is negative.

Dotted lines in Fig. 6] show the 1d energy per particle calculated within the LDA for $g_{1 d}^{0}$ (using the LL equation of state), while solid lines show the 1d energy per particle calculated within the LDA for $g_{1 d}$, Eq. (3) (using the LL equation of state for $g_{1 d}>0$, and the hard-rod equation of state for $\left.g_{1 d}<0\right)$. Remarkably, the LDA energies nearly coincide with the 1d many-body DMC energies calculated using the unrenormalized coupling constant (pluses) and the renormalized coupling constant (squares), respectively. Finite-size effects play a minor role only for $a_{3 d} \ll a_{\rho}$. Our calculations thus establish that a simple treatment, i.e., a hard-rod equation of state treated within the LDA, describes inhomogeneous quasi1d Bose gases with negative coupling constant $g_{1 d}$ well over a wide range of $3 \mathrm{~d}$ scattering lengths $a_{3 d}$.

For $a_{3 d} \rightarrow 0^{-}$, that is, for large $a_{1 d}$, the hard-rod equation of state treated within the LDA, cannot properly describe trapped quasi-1d Bose gases, which are expected to become unstable against formation of cluster-like manybody bound states for $a_{1 d} \approx 1 / n_{1 d}$. Thus, Sec. VI investigates the regime with negative $a_{3 d}$ in more detail within a many-body framework.

\section{STABILITY OF QUASI-ONE-DIMENSIONAL BOSE GASES}

This section discusses the stability of inhomogeneous quasi-1d Bose gases with negative $g_{1 d}$, that is, with $a_{3 d}>a_{3 d}^{c}$ and $a_{3 d}<0$, against cluster formation. Section $\mathrm{VB}$ shows that the (FN-)DMC results for the $1 \mathrm{~d}$ Hamiltonian, Eq. (20), are in very good agreement with the FN-DMC results for the $3 \mathrm{~d}$ Hamiltonian. Hence, we carry our analysis out within the $1 \mathrm{~d}$ model Hamiltonian, Eq. (20); we believe that our final conclusions also hold for the 3d Hamiltonian, Eq. (19). For the inhomogeneous 1d Hamiltonian $H_{1 d}$, Eq. (20), the lowest-lying gas-like state is a highly-excited state (see Sec. III). We now address the question whether this state is stable quantitatively using the VMC method.

We solve the 1d many-body Schrödinger equation for the Hamiltonian $H_{1 d}$, Eq. (20), by the VMC method using the trial wave function $\psi_{T}$ given by Eqs. (39) and 


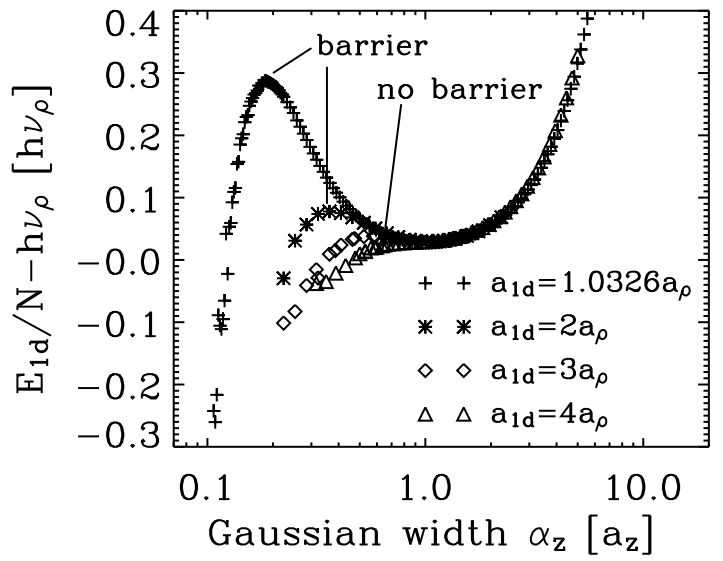

FIG. 7: VMC energy per particle, $E_{1 d} / N-\hbar \omega_{\rho}$, as a function of the variational parameter $\alpha_{z}$ for $N=5, \lambda=0.01$ and $a_{1 d} / a_{\rho}=1.0326$ (pluses), 2 (asterisks), 3 (diamonds) and 4 (triangles). An energy barrier is present for $a_{1 d} / a_{\rho}=1.0326$ and 2 , but not for $a_{1 d} / a_{\rho}=4$.

(40). This many-body wave function has the same nodal constraint as a system of $N$ hard-rods of size $a_{1 d}$. However, contrary to hard-rods, for interparticle distances smaller than $a_{1 d}$ the amplitude of the wave function increases as $|z|$ decreases. This effect arises from the attractive nature of the $1 \mathrm{~d}$ effective potential and gives rise in the many-body framework to the formation of clusterlike bound states as the average interparticle distance is reduced below a certain critical value.

Figure 7 shows the resulting VMC energy per particle, $E_{1 d} / N-\hbar \omega_{\rho}$, for $N=5$ and $\lambda=0.01$ as a function of the Gaussian width $\alpha_{z}$ for four different values of $a_{1 d}$. For $a_{1 d} / a_{\rho}=1.0326$ and 2, Fig. 7 shows a local minimum at $\alpha_{z, \text { min }} \approx a_{z}$. The minimum VMC energy nearly coincides with the FN-DMC energy (see also Fig. 8), which suggests that our variational wave function provides a highly accurate description of the quasi-1d many-body system. The energy barrier at $\alpha_{z} \approx 0.2 a_{z}$ decreases with increasing $a_{1 d}$, and disappears for $a_{1 d} / a_{\rho} \approx 3$. We interpret this vanishing of the energy barrier as an indication of instability 31]. For small $a_{1 d}$, the energy barrier separates the lowest-lying gas-like state from cluster-like bound states. Hence, the gas-like state is stable against cluster formation. For larger $a_{1 d}$, this energy barrier disappears and the gas-like state becomes unstable against cluster formation.

We stress that our stability analysis should not be confused with that carried out for attractive inhomogeneous $3 \mathrm{~d}$ systems at the level of mean-field Gross-Pitaevskii theory [32]. In fact, a mean-field type analysis of inhomogeneous 1d Bose gases does not predict stability of gas-like states [33. In our analysis, the emergence of local energy minima in configuration space is due to the structure of the two-body correlation factor $f_{2}(z)$ entering the VMC

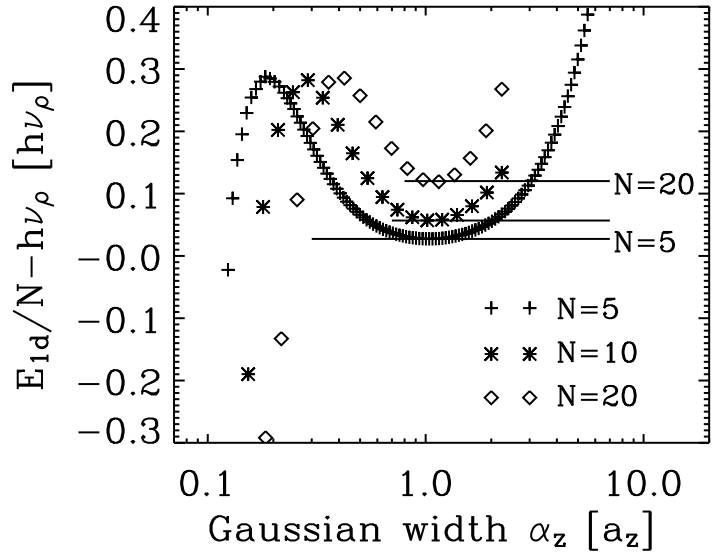

FIG. 8: VMC energy per particle, $E_{1 d} / N-\hbar \omega_{\rho}$, as a function of the variational parameter $\alpha_{z}$ for $a_{1 d} / a_{\rho}=1.0326$ (corresponding to the unitary regime), $\lambda=0.01$, and $N=5$ (pluses), 10 (asterisks), and 20 (diamonds). (The $N=5$ data are also shown in Fig. 7) The height of the energy barrier decreases with increasing $N$. Horizontal solid lines show the corresponding energies for $N=5,10$ and 20 obtained using the FN-DMC technique, which are in excellent agreement with the VMC energy obtained for $\alpha_{z}=\alpha_{z, \min }$.

trial wave function $\psi_{T}$, Eqs. (39) and (40). It is a manybody effect that cannot be described within a mean-field Gross-Pitaevskii framework.

To additionally investigate the dependence of stability on the number of particles, Fig. [ 8 shows the VMC energy for $\lambda=0.01$ as a function of the variational parameter $\alpha_{z}$ for different values of $N, N=5,10$ and 20. The scattering length $a_{1 d}$ is fixed at the value corresponding to the unitary regime, $a_{1 d}=1.0326 a_{\rho}$. Figure 8 shows that the height of the energy barrier decreases for increasing $N$. Figures 7 and 8 suggest that the stability of $1 \mathrm{~d}$ Bose gases depends on $a_{1 d}$ and $N$. To extract a functional dependence, we additionally perform variational calculations for larger $N$ and different values of $\lambda$ and $a_{1 d}$. We find that the onset of instability of the lowest-lying gas-like state can be described by the following criticality condition

$$
\sqrt{N \lambda} \frac{a_{1 d}}{a_{\rho}} \simeq 0.78,
$$

or, equivalently, by $\sqrt{N} a_{1 d} / a_{z} \simeq 0.78$. Our $1 \mathrm{~d}$ manybody calculations thus suggest that the lowest-lying gaslike state is stable if $\sqrt{N \lambda} a_{1 d} / a_{\rho} \lesssim 0.78$, and that it is unstable if $\sqrt{N \lambda} a_{1 d} / a_{\rho} \gtrsim 0.78$. The stability condition, Eq. (46), implies that reducing the anisotropy parameter $\lambda$ should allow stabilization of relatively large quasi-1d Bose gases.

To express the stability condition, Eq. (46), in terms of the $1 \mathrm{~d}$ gas parameter $n_{1 d} a_{1 d}$, where $n_{1 d}$ denotes the linear density at the trap center, we approximate the density 


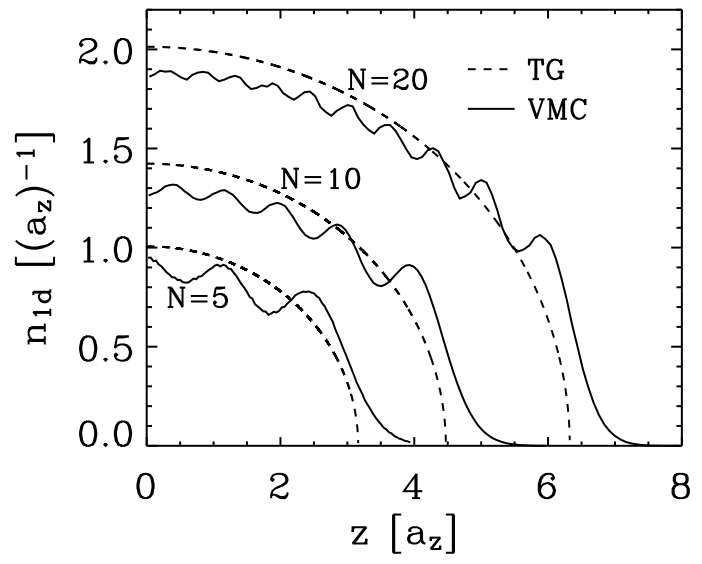

FIG. 9: TG density [Eq. (26), dashed lines] as a function of $z$ together with VMC density (solid lines), obtained by solving the 1d many-body Schrödinger equation, Eq. (22), for $N=5$ and $a_{1 d} / a_{\rho}=3.6$, for $N=10$ and $a_{1 d} / a_{\rho}=2.6$, and for $N=20$ and $a_{1 d} / a_{\rho}=1.8$. The TG density at the center of the trap, $z=0$, deviates from the VMC density at the center of the trap by less than $10 \%$.

for negative $g_{1 d}$ by the TG density, Eq. 26). Figure 9 compares the TG density with that obtained from the VMC calculations for $N=5,10$ and 20 and values of $a_{1 d} / a_{\rho}$ close to the criticality condition, Eq. (46). The density at the center of the trap is described by the TG density to within $10 \%$. Since the TG density at the trap center is given by $\sqrt{2 N} /\left(\pi a_{z}\right)$ [see Eq. (26)], the stability condition, Eq. (46), expressed in terms of the $1 \mathrm{~d}$ gas parameter reads $n_{1 d} a_{1 d} \lesssim 0.35$.

\section{CONCLUSIONS}

This paper presents a thorough study of the properties of inhomogeneous, harmonically-confined quasi-1d Bose gases as a function of the $3 \mathrm{~d}$ scattering length $a_{3 d}$. The behavior of confined Bose gases strongly depends on the ratio of the harmonic oscillator length in the tight transverse direction, $a_{\rho}$, to the interaction range $r_{0}$ and to the average interparticle distance $1 / n^{1 / 3}$, where $n$ denotes the $3 \mathrm{~d}$ central density.

Quasi-1d bosonic gases have been realized experimentally in highly-elongated harmonic traps. The strength of atom-atom interactions can be varied over a wide range by tuning the value of the $3 \mathrm{~d} s$-wave scattering length $a_{3 d}$ through application of an external magnetic field in the proximity of a Feshbach resonance. For $r_{0} \ll a_{\rho}$, the scattering length $a_{3 d}$ determines to a good approximation the effective $1 \mathrm{~d}$ scattering length $a_{1 d}$ and the effective $1 \mathrm{~d}$ coupling constant $g_{1 d}$, which can be, just as the $3 \mathrm{~d}$ coupling constant, tuned to essentially any value including zero and $\pm \infty$. By exploiting Feshbach resonance tech- niques, one should be able to achieve strongly-correlated quasi-1d systems. The strong coupling regime is achieved for $1 / n^{1 / 3} \gg a_{\rho}$, it includes the TG gas, where a system of interacting bosons behaves as if it consisted of noninteracting spinless fermions, and the so-called unitary regime, where the properties of the gas become independent of the actual value of $a_{3 d}$. In the unitary regime, the gas is dilute, that is, $n r_{0}^{3} \ll 1$, and at the same time strongly-correlated, that is, $n\left|a_{3 d}\right|^{3} \gg 1$.

The present analysis is carried out within various theoretical frameworks. We obtain the $3 \mathrm{~d}$ energetics of the lowest-lying gas-like state of the system using a microscopic FN-DMC approach, which accounts for all degrees of freedom explicitly. The resulting energetics are then used to benchmark our 1d calculations. Full microscopic $1 \mathrm{~d}$ calculations for contact interactions with renormalized coupling constant $g_{1 d}$ result in energies that are in excellent agreement with the full $3 \mathrm{~d}$ energies. This agreement implies that a properly chosen many-body 1d Hamiltonian describes quasi-1d Bose gases well.

We also consider the LL and the hard-rod equation of state of a $1 \mathrm{~d}$ system treated within the LDA. These approaches provide a good description of the energy of the lowest-lying gas-like state for as few as five or ten particles. Finite size effects are to a good approximation negligible. Our detailed microscopic studies suggest that these LDA treatments provide a good description of quasi-1d Bose gases. In particular, we suggest a simple treatment of $1 \mathrm{~d}$ systems with negative $g_{1 d}$ using the hard-rod equation of state.

Finally, we address the question of whether the lowestlying gas-like state of inhomogeneous quasi-1d Bose gases is actually stable. We find, utilizing a variational $1 \mathrm{~d}$ many-body framework, that the lowest-lying gas-like state is stable for negative coupling constants, up to a minimum critical value of $\left|g_{1 d}\right|$. Our numerical results suggest that the stability condition can be expressed as $n_{1 d} a_{1 d} \simeq 0.35$. Since our conclusions are derived from variational $1 \mathrm{~d}$ calculations, more thorough microscopic calculations are needed to confirm our findings. We believe, however, that our findings will hold even in a $3 \mathrm{~d}$ framework or when three-body recombination effects are included explicitly.

While our study was performed for inhomogeneous quasi-1d Bose gases, many findings also apply to homogeneous quasi-1d Bose gases. Furthermore, the Fermi-Bose mapping 3, 14, 34], which allows one to map an interacting $1 \mathrm{~d}$ gas of spin-polarized fermions to an interacting $1 \mathrm{~d}$ gas of spin-polarized bosons, suggests that many of the results presented here for quasi-1d Bose gases may directly apply to quasi-1d Fermi gases.

Acknowledgments: GEA and SG acknowledge support by the Ministero dell'Istruzione, dell'Università e della Ricerca (MIUR). DB acknowledges support by the NSF (grants 0331529 and 0218643) and generous hospitality by the BEC Center at the University of Trento. BEG acknowledges support by the NSF through a grant to ITAMP. 
[1] A. Görlitz et al., Phys. Rev. Lett. 87, 130402 (2001); F. Schreck et al., ibid. 87, 080403 (2001); M. Greiner et al., ibid. 87, 160405 (2001).

[2] L. Tonks, Phys. Rev. 50, 955 (1936).

[3] M. Girardeau, J. Math. Phys. 1, 516 (1960).

[4] M. Olshanii, Phys. Rev. Lett. 81, 938 (1998).

[5] D.S. Petrov, G.V. Shlyapnikov and J.T.M. Walraven, Phys. Rev. Lett. 85, 3745 (2000).

[6] H. Moritz et al., preprint cond-mat/0307607 J. Reichel and J. Thywissen, preprint cond-mat/0310330

[7] S. Inouye et al., Nature 392, 151 (1998); S.L. Cornish et al., Phys. Rev. Lett. 85, 1795 (2000); T Loftus et al., Phys. Rev. Lett. 88, 173201 (2002).

[8] K.M. O'Hara et al., Science 298, 2179 (2002); T. Bourdel et al., preprint cond-mat/0303079

[9] H. Heiselberg, Phys. Rev. A 63, 043606 (2001).

[10] S. Cowell et al., Phys. Rev. Lett. 88, 210403 (2002).

[11] T. Ho and E. Mueller, preprint cond-mat/0306187

[12] D.M. Gangardt and G.V. Shlyapnikov, Phys. Rev. Lett. 90, 010401 (2003).

[13] G.E. Astrakharchik et al., preprint cond-mat/0308585

[14] B.E. Granger and D. Blume, preprint cond-mat/0307358

[15] T. Busch et al., Found. Phys. 28, 549 (1998).

[16] E. Tiesinga, C.J. Williams, F.H. Mies, and P.S. Julienne, Phys. Rev. A 61, 063416 (2000).

[17] T. Bergeman, M.G. Moore and M. Olshanii, Phys. Rev. Lett. 91, 163201 (2003).

[18] E.L. Bolda, E. Tiesinga and P.S. Julienne, Phys. Rev. A 68, 032702 (2003).

[19] D. Blume, Phys. Rev. A 66, 053613 (2002); G. E. Astrakharchik and S. Giorgini, ibid. 66, 053614 (2002).
[20] L.D. Carr, C. Clark and W.P. Reinhardt, Phys. Rev. A 62, 063611 (2000).

[21] R. Kanamoto, H. Saito and M. Ueda, Phys. Rev. A 67, 115208 (2003).

[22] K.E. Strecker et al., Nature 417, 150 (2002); L. Khaykovich et al., Science 296, 1290 (2002).

[23] V. Dunjko, V. Lorent and M. Olshanii, Phys. Rev. Lett. 86, 5413 (2001).

[24] E.H. Lieb and W. Liniger, Phys. Rev. 130, 1605 (1963); E.H. Lieb, ibid. 130, 1616 (1963); C.N. Yang and C.P. Yang, J. Math. Phys. 10, 1115 (1969).

[25] J.B. McGuire, J. Math. Phys. 5, 622 (1964).

[26] R. Guardiola, in Microscopic Quantum Many-Body Theories and Their Applications, ed. by J. Navarro and A. Polls (Springer, Berlin, 1998).

[27] W.L. McMillan, Phys. Rev. 138, 442 (1965).

[28] see, e.g., J. L. DuBois and H. R. Glyde, Phys. Rev. A 63, 023602 (2001); G. E. Astrakharchik and S. Giorgini, ibid. 68, 031602(R) (2003).

[29] J. Boronat and J. Casulleras, Phys. Rev. B 49, 8920 (1994).

[30] P.J. Reynolds et al., J. Chem. Phys. 77, 5593 (1982).

[31] J.L. Bohn, B.D. Esry and C.H. Greene, Phys. Rev. A 58, 584 (1998).

[32] G. Baym and C. Pethick, Phys. Rev. Lett. 76, 6 (1996).

[33] L.D. Carr, J.N. Kutz and W.P. Reinhardt, Phys. Rev. E 63, 066604 (2001).

[34] T. Cheon and T. Shigehara, Phys. Rev. Lett. 82, 2536 (1999); M.D. Girardeau and M. Olshanii, preprint cond-mat/0309396 